\title{
A Nuclear Variant of ErbB3 Receptor Tyrosine Kinase Regulates Ezrin Distribution and Schwann Cell Myelination
}

\author{
Tadepalli Adilakshmi, ${ }^{1}$ Jennifer Ness-Myers, ${ }^{1}$ Carlos Madrid-Aliste, ${ }^{2,3}$ Andras Fiser, ${ }^{2,3}$ and Nikos Tapinos ${ }^{1}$ \\ ${ }^{1}$ Molecular Neuroscience and Regeneration Laboratory, Weis Center for Research, Geisinger Clinic, Danville, Pennsylvania 17822, and Departments of \\ ${ }^{2}$ Systems and Computational Biology and ${ }^{3}$ Biochemistry, Albert Einstein College of Medicine, Bronx, New York 10461
}

Reciprocal interactions between glia and neurons are essential for the proper organization and function of the nervous system. Recently, the interaction between ErbB receptors (ErbB2 and ErbB3) on the surface of Schwann cells and neuronal Neuregulin-1 (NRG1) has emerged as the pivotal signal that controls Schwann cell development, association with axons, and myelination. To understand the function of NRG1-ErbB2/3 signaling axis in adult Schwann cell biology, we are studying the specific role of ErbB3 receptor tyrosine kinase (RTK) since it is the receptor for NRG1 on the surface of Schwann cells. Here, we show that alternative transcription initiation results in the formation of a nuclear variant of ErbB3 (nuc-ErbB3) in rat primary Schwann cells. nuc-ErbB3 possesses a functional nuclear localization signal sequence and binds to chromatin. Using chromatin immunoprecipitation (ChIP)-chip arrays, we identified the promoters that associate with nuc-ErbB3 and clustered the active promoters in Schwann cell gene expression. nuc-ErbB3 regulates the transcriptional activity of ezrin and HMGB1 promoters, whereas inhibition of nuc-ErbB3 expression results in reduced myelination and altered distribution of ezrin in the nodes of Ranvier. Finally, we reveal that NRG1 regulates the translation of nuc-ErbB3 in rat Schwann cells. For the first time, to our knowledge, we show that alternative transcription initiation from a gene that encodes a RTK is capable to generate a protein variant of the receptor with a distinct role in molecular and cellular regulation. We propose a new concept for the molecular regulation of myelination through the expression and distinct role of nuc-ErbB3.

\section{Introduction}

ErbB3 receptor tyrosine kinase is a member of the ErbB receptor family that includes ErbB1 (EGFR), ErbB2 (Her2), ErbB3, and ErbB4 (Citri et al., 2003). During development, ErbB3 is found in cells of the gastrointestinal, reproductive, and urinary tracts, as well as the skin, endocrine, and nervous system (Prigent et al., 1992). Since ErbB3 lacks tyrosine kinase activity and ErbB2 is a ligandless receptor, both need to heterodimerize to be active. In the nervous system, the ligand for ErbB3 that induces ErbB2ErbB3 dimers and signaling activation is Neuregulin-1 (NRG1). Early studies showed that spinal cord motor neurons, dorsal root ganglion (DRG) sensory neurons, and autonomic neurons express NRG1 (Corfas et al., 1995) and that Schwann cells express ErbB2 and ErbB3 (Meyer and Birchmeier, 1995). The importance of NRG1-ErbB2/3 signaling in Schwann cell development has been demonstrated in vivo with genetically modified mice (Lee et al., 1995; Riethmacher et al., 1997; Morris et al., 1999; Woldeyesus et al., 1999). In ErbB3 knock-out mice, Schwann cells fail to

\footnotetext{
Received Oct. 27, 2010; revised Jan. 5, 2011; accepted Feb. 4, 2011

This work was supported by internal funds of the Geisinger Clinic and by National Institute of Neurological Disorders and Stroke Grant R01 NS070975 (N.T.). We thank Dr. Marius Sudol for providing the STREP-ErbB4 FL plasmid and Dr. Steven Toms for the FLICA Apoptosis Detection reagent. We express our appreciation to Dr. David Carey and the Weis Center Staff for continuous support. All animal work was performed according to institutional guidelines.

The authors declare no authorship-related conflict of interest and no competing financial interests.

Correspondence should be addressed to Dr. Nikos Tapinos, Molecular Neuroscience and Regeneration

Laboratory, Weis Center for Research, Geisinger Clinic, 100 North Academy Avenue, Danville, PA 17822. E-mail: ntapinos1@geisinger.edu.

DOI:10.1523/JNEUROSCI.5635-10.2011

Copyright $\odot 2011$ the authors $\quad 0270-6474 / 11 / 315106-14 \$ 15.00 / 0$
}

develop, and most sensory and motor neurons die subsequently (Davies, 1998). Signaling through the neuregulin/ErbB2-3 axis has been implicated in the regulation of Schwann cell myelination (Garratt et al., 2000; Michailov et al., 2004; Taveggia et al., 2005) migration and axonal sorting (Yamauchi et al., 2008).

In an effort to characterize the role of ErbB3 in the molecular processes that regulate myelination, we noticed consistent presence of ErbB3 in the nucleus of Schwann cells. Recently, nuclear localization of ErbB3 has been reported in Schwann cells (Raabe et al., 2004), mammary epithelial cells (Offterdinger et al., 2002), and prostate cancer cells (Koumakpayi et al., 2006, 2007). However, neither the precise function nor the mechanism of nuclear localization of ErbB3 is understood. Here, we identify a novel nuclear variant of ErbB3 (nuc-ErbB3) that is produced through alternative transcription initiation and encodes part of the cytoplasmic domain of the full-length ErbB3. We show that the translation rate and expression of nuc-ErbB3 in Schwann cells depends on neuregulin signaling. nuc-ErbB3 is capable of nuclear localization because of a functional nuclear localization sequence (NLS) motif. nuc-ErbB3 binds to chromatin and associates with genomic regions that include promoters of genes, which are expressed in Schwann cells. One of these genes is ezrin that has been shown to be a component of Schwann cell microvilli with a role in the formation of the nodes of Ranvier (Melendez-Vasquez et al., 2001; Scherer et al., 2001). nuc-ErbB3 regulates the promoter activity of ezrin and affects the distribution of ezrin in the Schwann cell microvilli that participate in the proper formation of the nodes of Ranvier. Finally, we show that nuc-ErbB3 regulates extent of myelination by Schwann cells. 


\section{Materials and Methods}

Purification of primary rat Schwann cells. Purification of rat primary Schwann cells from the sciatic nerves of female and male postnatal day 2 (P2) pups was described previously (Einheber et al., 1993).

Rat Schwann cell-neuron cocultures. Isolation and culturing of rat dorsal root ganglion neurons, Schwann cell coculture, and myelination protocols were described previously (Carey and Todd, 1987).

Other cell lines. Cos-7 cells were purchased from American Type Culture Collection and cultured in DMEM supplemented with 10\% FBS (HyClone).

Antibodies. Primary antibodies used in this study included rabbit anti-ErbB3 (C-terminal specific) (Santa Cruz), rabbit anti-ErbB3 (Nterminal specific) (Calbiochem), mouse anti-ErbB3 (RTJ-2 clone) (Santa Cruz; Abcam), rabbit anti-ErbB2 and anti-ErbB3 (Santa Cruz), mouse anti-ErbB3 (Abcam), rabbit anti-ErbB4 (Santa Cruz), mouse antiATPase (Abcam), mouse anti- $\beta$-actin (Sigma-Aldrich), rabbit antiLamin A (Abcam), rabbit anti-pan-neuronal neurofilament (Covance), mouse anti-myelin basic protein (MBP) (Covance), rabbit anti-Ezrin (Cell Signaling), rabbit anti-eIF4E (Santa Cruz), and rabbit anti-S-100 (Dako).

Plasmids and transfections. Full-length rat ErbB3 (nucleotides 1-4158) and nuc-ErbB3 were amplified by PCR from rat Schwann cell cDNA and cloned into vectors pcDNA 3.1 and pcDNA 3.1 TOPO, respectively (Invitrogen). To study the mechanism of nuclear translocation of nucErbB3, a point mutation was introduced in the NLS region of the nucErbB3 using QuikChange mutagenesis kit (Stratagene) following the manufacturer's instructions. Correct orientation and sequence integrity of each construct was verified by sequencing. Human cDNA clones of full-length ErbB2 and ErbB3 were purchased from Open Biosystems. Each of the above-mentioned constructs were introduced into confluent $(\sim 85 \%)$ four-well dishes of Cos-7 cells using Fugene HD (Roche) according to the manufacturer's instructions. Two days after transfection, cells were fixed and stained with ErbB3 and ErbB2 antibodies and visualized with a Zeiss Axiovert inverted microscope equipped with a highresolution digital camera. For cotransfection of ErbB2 and ErbB3, the plasmids were used in equimolar amounts. Membrane localization of ErbB2/ErbB3 receptors and ErbB3 transphosphorylation was induced with $\beta 1$-heregulin (R\&D Systems) treatment of Cos-7 cells for 15 min. Subsequently, ErbB3 receptor activation was monitored by immunoprecipitation of lysates with ErbB3 antibody before and after the addition of $\beta 1$-heregulin and blotting the precipitates with phosphotyrosine antibody.

Immunofluorescence. Cells were fixed with $4 \%$ formaldehyde and permeabilized with $0.5 \%$ Triton X-100 or methanol. After blocking and incubation with the relevant primary antibodies, the cells were washed and incubated with affinity-purified, Alexa Fluor 488/594-conjugated goat anti-mouse/goat anti-rabbit IgG (Invitrogen), respectively. Images were captured with a Zeiss Axiovert inverted microscope equipped with Apotome and a high-resolution digital camera.

5-Bromodeoxyuridine and FLICA labeling. Cells were transfected with small interfering RNA (siRNA) constructs and grown for $3 \mathrm{~d}$. Cells were then incubated with 5-bromodeoxyuridine (BrdU) for $1 \mathrm{~h}$, fixed with $70 \%$ ethanol in $50 \mathrm{~mm}$ glycine, $\mathrm{pH} 2$, and $\mathrm{BrdU}$ immunostaining was done according to manufacturer's directions (Roche). The FLICA substrate (a kind gift from Dr. Steve Toms, Geisinger Clinic, Danville, PA) was used to detect caspase activity in cells $3 \mathrm{~d}$ after transfection with siRNA constructs. After $1 \mathrm{~h}$ incubation with the FLICA substrate, cells were stained with propidium iodide. Images were captured with a Zeiss Axiovert inverted microscope.

Cell fractionation. Cells $\left(1 \times 10^{6}\right)$ were lysed by incubating buffer containing 20 mм Tris-HCl, pH 7.4, $150 \mathrm{~mm} \mathrm{KCl,} 1.5 \mathrm{~mm} \mathrm{MgCl}_{2}, 100 \mathrm{~mm}$ $\mathrm{NaF}$, protease and phosphatase inhibitors (Sigma), $1 \mathrm{~mm}$ phenylmethylsulfonylfluoride, $1 \mathrm{~mm}$ DTT, and $0.5 \%$ Nonidet P- 40 on ice. Cytoplasmic and nuclear fractions were separated by centrifugation at $1500 \times g$ for 5 $\min$ at $4^{\circ} \mathrm{C}$. The nuclear pellet was resuspended in the lysis buffer for an additional $20 \mathrm{~min}$ to ensure complete lysis of any residual cells and centrifuged. The resulting nuclei were washed twice in buffer A $(10 \mathrm{~mm}$ HEPES, pH 7.9, $10 \mathrm{~mm} \mathrm{KCl,} 1.5 \mathrm{~mm} \mathrm{Mg}_{2} \mathrm{Cl}, 0.34 \mathrm{~m}$ sucrose, and $10 \%$ glycerol) and centrifuged. The nuclei were then lysed in no salt buffer ( 3
mM EDTA, 0.2 mu EGTA) for $30 \mathrm{~min}$ on ice with frequent vortexing. The nuclear soluble protein was separated by centrifugation at $2000 \times g$ for 5 $\min$ at $4^{\circ} \mathrm{C}$. The pellet serves as the chromatin fraction.

Incubation of Schwann cells with various inhibitors of cleavage pathways. Serum and growth factor-starved Schwann cells were treated for $1 \mathrm{~h}$ with 10 nм (2R)- $N^{\prime}$-hydroxy- $N$-[(2S)-3-(5H-indol-3-yl)-1-methylamino-1-oxopropan-2-yl]-2-(2-methylpropyl)butanediamide (GM6001) (metalloprotease inhibitor) (Calbiochem), or $N$-(3, 5-difluorophenacetyl-L-alanyl)-Sphenylglycine $t$-butyl ester (IX) ( $\gamma$-secretase) (Calbiochem), or ZLL2 (site-2 protease) (Calbiochem), or DIC (Rhomboid) (Calbiochem), or zVADFMK (caspase) (Promega), or lactacystin (proteasome) (Sigma-Aldrich). Subsequently, the cells were treated with $5 \mu \mathrm{g} / \mathrm{ml} \beta 1$-heregulin with $10 \mu \mathrm{M}$ of the respective inhibitors for $30 \mathrm{~min}$, and the cells were fixed and processed for immunofluorescence to examine the putative inhibition of the ligandinduced nuclear translocation of ErbB3.

Sequence analysis for metalloprotease recognition site in ErbB3. Rat Schwann cell ErbB3 was aligned with ErbB4 and its isoforms differing in the juxtamembrane regions, JM-a and JM-b, using MacVector 10.5.

Reverse transcription-PCR. Total RNA $(10 \mu \mathrm{g})$ from rat primary Schwann cells or rat sciatic nerves was treated with DNaseI and purified by phenol/chloroform extraction and ethanol precipitation. First-strand cDNA was synthesized from purified RNA $(5 \mu \mathrm{g})$ using Superscript III $\mathrm{RT}$ (Invitrogen) at $50^{\circ} \mathrm{C}$ for $60 \mathrm{~min}$. cDNA was treated with $\mathrm{RNaseH}$ to remove residual RNA and an aliquot was used for PCR amplification of nuc-ErbB3 (1-2 $\mu \mathrm{l})$.

The following primers were used to amplify the $\sim 1500$ bp region of nucErbB3 starting from the $5^{\prime}$ - untranslated region (UTR) and ending at the last exon of the ErbB3 sequence excluding all introns, and the two internal exonic regions amplified by nested PCR ( $\sim 500$ and $\sim 350 \mathrm{bp}): 5^{\prime}$-UTR forward (product 1), TTTTTCGGAGCTGGGGATCG; nuc-ErbB3 reverse (product 1), TTTTGAGGCGGGCATAACG; nuc-ErbB3-exon forward (product 2), CCACTTTGGGAAATACACAC; nuc-ErbB3-exon reverse (product 2), CCAGATGAGGGACTTAA; nuc-ErbB3-exon forward (product 3), CATGCCCATGAACCAGAGCA; nuc-ErbB3-exon reverse (product 3), CCTCCTCAGTACCCAACACA.

Northern blotting. mRNA was purified from total Schwann cell RNA using Fast Track MAG mRNA isolation kit (Invitrogen). One microgram of mRNA was denatured and separated on a $1 \%$ formaldehyde denaturing gel and transferred to Hybond-N+ membrane using Genie electroblotter (Idea Scientific). After cross-linking RNA to membrane by UV at $120 \mathrm{~mJ} / \mathrm{cm}^{2}$ (VWR UVP), the membrane was prehybridized and hybridized with a biotin-labeled ErbB3 cytoplasmic domain-specific RNA probe at $65^{\circ} \mathrm{C}$ for overnight. Stringency washes, streptavidin binding, and signal detection were performed according to manufacturer's instructions (Bright Star Biodetect; Ambion). Multiple tissue mRNA blot was purchased from Ambion. Northern blotting was performed using biotinylated ErbB3-specific RNA probe and was developed using manufacturer's instructions (BrightStar Psoralen-Biotin kit and BrightStar Biodetect kit, respectively, from Ambion).

Prediction of NLS and ErbB3 expression topology. To identify a potential NLS in the ErbB3 protein sequence we used the PredictProtein portal (www.predictprotein.org/) that compares the input sequence with multiple protein sequences containing experimentally identified NLS sequences (Cokol et al., 2000). Prediction of ErbB3 expression topology and DNA binding was performed through the DBS-PRED portal (Ahmad et al., 2004) and the SubLoc, version 1.0, portal: http://www.bioinfo.tsinghua.edu.cn/.

Chromatin immunoprecipitation-chip array. For the chromatin immunoprecipitation (ChIP)-chip array, we performed the ChIP protocol of Weinmann and Farnham (2002) with slight modifications. Briefly, $1 \times 10^{8}$ rat primary Schwann cells were cross-linked with $1 \%$ formaldehyde followed by the addition of $0.125 \mathrm{~m}$ glycine to stop the reaction. Subsequently, the cells were trypsinized, resuspended in cell lysis buffer consisting of 5 mм PIPES, pH 8.0, $85 \mathrm{~mm} \mathrm{KCl,} \mathrm{0.5 \%} \mathrm{Nonidet} \mathrm{P-40,} \mathrm{and}$ protease inhibitor mixture (Sigma-Aldrich). The cell lysate was centrifuged, and the pellet was resuspended in SDS lysis buffer. The chromatin was then sonicated to an average length of $1-2 \mathrm{~kb}$ on ice using a Misonix 3000 sonicator and centrifuged. The supernatant containing the chromatin was carefully removed and precleared with protein A beads (Pierce). 
For the nuc-ErbB3 immunoprecipitation, we used the rabbit anticErbB3 antibody (Santa Cruz) and protein A beads (Pierce). Subsequently, the histone complex was eluted from the antibody with $1 \%$ SDS, $0.1 \mathrm{M} \mathrm{NaHCO}_{3}$ (elution buffer) under constant rotation for $15 \mathrm{~min}$ at room temperature. To reverse the chromatin/DNA cross-links, we treated the elutions with $5 \mathrm{M} \mathrm{NaCl}$ for $4 \mathrm{~h}$ at $65^{\circ} \mathrm{C}$ and then amplified using the whole genome amplification (WGA) kit from Sigma-Aldrich according to the manufacturer's instructions. Subsequently, the samples were submitted to Roche/NimbleGen for additional quality evaluations, hybridizations, and analysis. For our experiments, we used the rat ChIP $385 \mathrm{~K}$ promoter set in duplicates using a no-antibody ChIP control for normalization and background extraction.

Luciferase assays. Luciferase assays were performed using the SteadyGlo Luciferase Assay (Promega) according to the manufacturer's instructions. The ezrin promoter/luciferase construct was purchased from SwitchGear Genomics. Transfection efficiencies were normalized against the expression of Renilla Luciferase (Promega).

eIF4E immunoprecipitation and reverse transcription-PCR. Immunopurification and reverse transcription (RT)-PCR were according to Ishigaki et al. (2001). Briefly, rat Schwann cells $\left(4 \times 10^{7}\right)$ that were treated with $5 \mathrm{ng} / \mathrm{ml}$ heregulin for $30 \mathrm{~min}$ or untreated controls were washed three times with ice-cold PBS. Cells were harvested and suspended in PBS. After centrifugation, cell pellets were resuspended in $0.5 \mathrm{ml}$ of NET-2 buffer (50 mм Tris-HCl, pH 7.4, 300 mм NaCl, 0.05\% NP-40) containing $100 \mathrm{U}$ of RNase inhibitor (Invitrogen). Cells were sonicated with three $10 \mathrm{~s}$ bursts using a Branson Sonifier. After sonication, homogenates were centrifuged at $10,000 \times g$ for $10 \mathrm{~min}$ at $4^{\circ} \mathrm{C}$. The supernatants were used as the source of antigen in the immunopurifications. They were first cleared by incubation with end-over-end rotation in the presence of $50 \mu$ l of protein A/G-agarose beads (Santa Cruz) for $30 \mathrm{~min}$ at $4^{\circ} \mathrm{C}$ followed by centrifugation at $10,000 \times g$. Cleared supernatants were then rotated in the presence of eIF4E antiserum (Santa Cruz) or normal rabbit serum as control for $90 \mathrm{~min}$ at $4^{\circ} \mathrm{C}$, after which $0.5 \mathrm{mg}$ of yeast tRNA (Sigma-Aldrich), $100 \mathrm{U}$ of RNase inhibitor, and protein A-agarose beads were added. The incubation was continued for another $60 \mathrm{~min}$ at $4^{\circ} \mathrm{C}$. The beads were washed six times with NET-2 buffer, suspended in $50 \mu \mathrm{l}$ of SDS sample buffer $(0.1$ м Tris- $\mathrm{HCl}, \mathrm{pH} 6.8,4 \%$ SDS, $20 \%$ glycerol, $12 \%$ 2-mercaptoethanol), and split into two portions. One portion was used as a source of protein, which was analyzed by SDS-PAGE and Western blotting. The other portion was extracted with phenol, chloroform, and isoamyl alcohol, and precipitated using ethanol. Precipitates were dissolved in $10 \mu \mathrm{l}$ of NET-2 buffer, treated with $10 \mathrm{U}$ of DNase I (Promega) for $15 \mathrm{~min}$ at $37^{\circ} \mathrm{C}$, extracted, precipitated, dissolved in $10 \mu \mathrm{l}$ of water, and used as a source of RNA for RT-PCR. Full-length and nucErbB3 were amplified from cDNA using specific primers.

Functional analysis of ErbB3 variants with siRNA interference. siRNAs were designed (Invitrogen) against a region of the $5^{\prime}$-UTR of nuc-ErbB3 (nucleotides 255-279), which corresponds to the intron between exons 22-23 of the full-length ErbB3 gene. We were not able to design any other siRNA that targets the $5^{\prime}$-UTR and shows selectivity for nuc-ErbB3, so we designed siRNAs against two regions of ErbB3 (nucleotides 263-288 and 3437-3462) such that the siRNA targeting the $\mathrm{N}^{\prime}$-terminus of ErbB3 between nucleotides 263-288 (full-length ErbB3-siRNA) would not interfere with the nuc-ErbB3, whereas nuc-ErbB3 siRNA would downregulate both forms (region between 3437 and 3462). Transfection of rat Schwann cells was optimized using a Nucleofector device and nucleofection optimization kit (Amaxa). Each siRNA (400 nM) was transfected into $1.0 \times 10^{6}$ rat Schwann cells using nucleofection (Amaxa). Transiently transfected cells were plated in Schwann cell media in six-well plates for $3 \mathrm{~d}$. This transfection protocol gave $60-70 \%$ efficiency as determined by flow cytometry. Finally, the siRNA-transfected cells were seeded on 3-week-old rat DRG neurons, and myelination was compared with that of nontransfected Schwann cells using fluorescence microscopy.

Statistical analysis of myelination. Myelination was induced by the addition of ascorbic acid ( $50 \mu \mathrm{g} / \mathrm{ml})$ and cultures were maintained in ascorbic acid for $14 \mathrm{~d}$. The cultures were then fixed and stained with MBP and Ezrin antibodies for detecting myelin and nodes, respectively, counterstained with 4',6-diamidino-2-phenylindole (DAPI), and visualized with
Axiocam (Zeiss). For analysis, photographs of the entire culture disc were taken at $10 \times$ magnification. The slides were selected randomly and myelin fragments (internodes) were counted blind. SD was calculated from three independent experiments, and the results were plotted on a histogram. Finally, a paired Student $t$ test was performed, and the results were considered significant when $p<0.05$.

\section{Results}

\section{Nuclear localization of ErbB3 in Schwann cells}

To examine the expression pattern of ErbB3, we stained purified cultures of rat primary Schwann cells with ErbB3 antibodies that recognize the C-terminal end of the intracellular domain (Santa Cruz) or a distinct epitope (RTJ-2 clone) at the cytoplasmic domain of ErbB3 (Abcam; Santa Cruz) (C-ErbB3). In addition, we used an antibody that recognizes the extracellular domain $(\mathrm{N}-$ ErbB3) of ErbB3 (Calbiochem). Whereas both C-ErbB3 antibodies stained the nucleus, cytoplasm, perinuclear region, and the plasma membrane of Schwann cells, N-ErbB3 stained only the membrane, perinuclear region, and cytoplasm (Fig. $1 A$ ), suggesting that the intracellular ErbB3 domain alone translocates to the nucleus of Schwann cells. Western blotting on total Schwann cell lysates with two antibodies that recognize distinct epitopes of the cytoplasmic domain of ErbB3 revealed several discrete bands of various molecular weights (e.g., 120, 80, and $50 \mathrm{kDa}$ ) in addition to the full-length ErbB3, which appears as a doublet at $\sim 180 \mathrm{kDa}$ as previously described (Xue et al., 2006; Lallemand et al., 2009) (Fig. $1 B$ ). We consider these bands to be ErbB3 specific since preincubation with an ErbB3 inhibitory peptide resulted in abrogation of all bands (Fig. $1 B$ ). To identify the putative ErbB3 fragment, which is present in the nucleus of Schwann cells, we prepared cytoplasmic and nuclear extracts from $1 \times 10^{7}$ Schwann cells and performed Western blotting with an antibody that recognizes the cytoplasmic domain of ErbB3 (Santa Cruz). This revealed the presence of the $180 \mathrm{kDa}$ full-length ErbB3 band exclusively in the cytoplasmic extracts and the presence of the $\sim 50 \mathrm{kDa}$ band exclusively in the nuclear extracts (Fig. 1C). The absence of the 120 and $80 \mathrm{kDa}$ bands from both extracts suggests that these correspond either to membrane-bound fragments or to nonspecific degradation products of ErbB3. Our argument regarding these ErbB3-reacting bands is based on the fact that we use hypotonic buffers to break open the cells, which release the soluble cytoplasmic proteins and the intact nuclei but not the membrane proteins that are discarded during the centrifugation. Also, the nuclei that were used to generate our nuclear preparation were subjected to sucrose cushions and extensive washes and monitored under the microscope to make sure that they were devoid of membranous threads that would eventually contaminate the nuclear fragment with non-nuclear proteins. The relatively low level of detectable ErbB3 in Schwann cell nuclear extracts and the very low level of the $50 \mathrm{kDa}$ band in total extracts (1/50th of the full-length ErbB3 band as quantified by densitometry) (Fig. $1 B, C$ ) may explain why this fragment was not reported previously. The complete absence of the full-length $\sim 180 \mathrm{kDa}$ ErbB3 from the nucleus suggests that only a portion of the cytoplasmic domain of ErbB3 is expressed in the nucleus of Schwann cells.

\section{The nuclear fragment of ErbB3 is not generated by proteolytic cleavage}

Nuclear expression of APP (amyloid precursor protein) (Cao and Sudhof, 2001), Notch (Steiner and Haass, 2000), ErbB4 (Ni et al., 2001), and p75 (Frade, 2005) has been noted in various cells, suggesting a conserved mechanism for membrane protein nuclear 
A

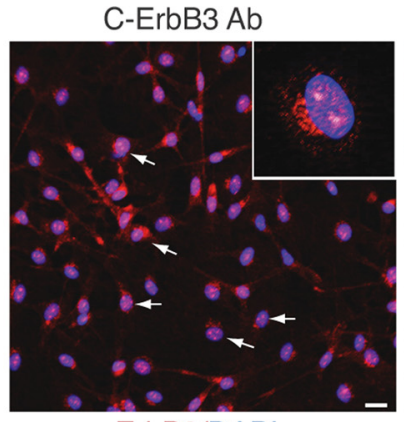

ErbB3/DAPI

B

Total Schwann cell lysates

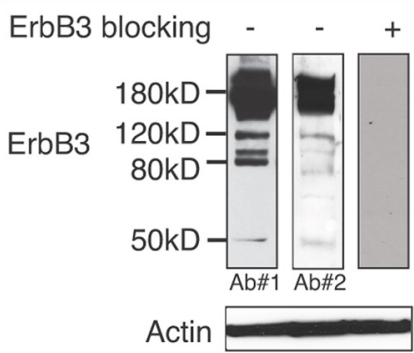

C

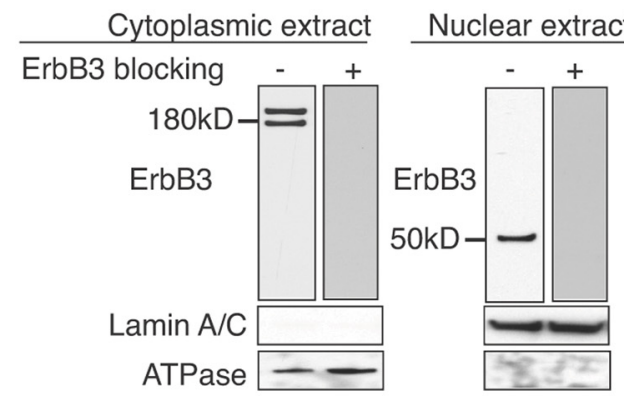

Figure 1. A $50 \mathrm{kDa}$ fragment of the cytoplasmic domain of ErbB3 is expressed in the nucleus of Schwann cells. $\boldsymbol{A}$, Immunostaining of purified rat primary Schwann cells with ErbB3 antibodies specific for the intracellular domain (C-ErbB3) or the extracellular domain (N-ErbB3) of ErbB3. Both pictures represent median images at the level of the nucleus from z-stacks (12 $\mu \mathrm{m}$ total with 2 $\mu \mathrm{m}$ increments) at $20 \times$ magnification. (-ErbB3 displayed staining in Schwann cell cytoplasm, membrane, perinuclear region, and nucleus (inset shows distinct pattern of speckled nuclear staining at $60 \times$ ), whereas N-ErbB3 staining was restricted to the membrane, cytoplasm, and perinuclear region (inset shows lack of nuclear staining at $60 \times$ ). $\boldsymbol{B}$, Total protein lysates of rat primary Schwann cells blotted with two antibodies against separate epitopes of the cytoplasmic domain of ErbB3. This reveals the fulllength ErbB3 band at $180 \mathrm{kDa}$ and additional bands at 120,80, and $50 \mathrm{kDa}$. Incubation with an ErbB3 blocking peptide abrogates all bands, suggesting that these are ErbB3 specific. Densitometric analysis from three independent experiments shows that the 50 $\mathrm{kDa}$ band corresponds to $\sim 1 / 50$ th of the density of the full-length ErbB3 band. $\beta$-Actin was used a loading control antibody. $\boldsymbol{C}$, Protein lysates of Schwann cells were separated into cytoplasmic and nuclear fractions and blotted with an antibody that recognizes the cytoplasmic portion of ErbB3. This revealed the existence of the full-length ErbB3 receptor ( $\sim 180 \mathrm{kDa}$ band) in the cytoplasmic fraction (left panel) and the $\sim 50 \mathrm{kDa}$ band in the nuclear fraction (right panel). The purity of the cytoplasmic and nuclear fractions was confirmed by the expression of ATPase and Lamin A/C in the corresponding lysates. Specificity of the ErbB3 antibody is verified with preincubation with an ErbB3 blocking peptide, which eliminates antibody binding in both the cytoplasmic and the nuclear lysates.

signaling. In all cases but ErbB3, an ectodomain cleavage by metalloproteases such as TACE/ADAM17 followed by a second transmembrane cleavage by $\gamma$-secretase has been proposed. This proteolytic cleavage pathway generates a free cytoplasmic domain portion of the respective protein, which translocates to the nucleus to participate in transcriptional regulation. Interestingly, the existence of two splice variants differing in the juxtamembrane cleavage recognition region has been reported in the case of ErbB4 (Elenius et al., 1997). Whereas the first variant (JMa-ErbB4) can be cleaved by metalloproteases, the second variant (JMb-ErbB4) lacks the cleavage recognition motif and cannot be cleaved. Sequence comparison of the juxtamembrane region of ErbB3 with both cleavable (JM-a) and noncleavable (JM-b) isoforms of ErbB4 revealed that ErbB3 resembles JMb-ErbB4 in that it lacks the specific metalloprotease amino acid recognition motif (Fig. $2 A$ ). We examined whether ErbB3 is subject to regulated intramembrane proteolysis in Schwann cells to produce the $50 \mathrm{kDa}$ nuclear variant and whether this depends on ligand activation. Schwann cells that had been serum and growth factor starved were treated with GM6001 (metalloprotease inhibitor) (Calbiochem), IX ( $\gamma$-secretase) (Calbiochem), ZLL2 (site-2 protease) (Calbiochem), and DIC (Rhomboid) (Calbiochem). Subsequently, $\beta 1$-heregulin was added in the presence of

the inhibitors, and then cells were fixed and stained for ErbB3 expression using a cytoplasmic specific ErbB3 antibody (Fig. 2B). These experiments revealed that none of the above inhibitors influence the expression of ErbB3 in the nucleus, indicating that ligand-mediated proteolytic cleavage may not be responsible for nuclear ErbB3 expression. They also confirm a previous report that ErbB3 cannot be cleaved by metalloproteases or $\gamma$-secretase in T47D carcinoma cells (Ni et al., 2001). The same results were obtained with zVAD-FMK (caspase) (Promega) or lactacystin (proteasome) (SigmaAldrich) inhibitors (data not shown). To verify the functionality of the protease inhibitors, we transfected Cos-7 cells with STREP-ErbB4-FL construct (a kind gift from Dr. Sudol, Geisinger Clinic, Danville, PA) and treated with the protease inhibitors as a positive control (supplemental data, available at www.jneurosci.org as supplemental material). This showed complete inhibition of the nuclear localization of ErbB4 (supplemental Fig. 1, available at www. jneurosci.org as supplemental material). To further confirm the absence of intramembrane proteolytic cleavage, we reconstituted the ErbB2-ErbB3 receptor tyrosine kinases (RTKs) in naive ErbB free cells. Cos-7 cells were cotransfected with equimolar amounts of full-length human ErbB2 and ErbB3 constructs (Open Biosystems). Immunoprecipitation with anti-ErbB3 antibody followed by phosphotyrosine Western analysis of the total lysates isolated from transfected Cos-7 cells after $\beta 1$-heregulin activation showed the phosphorylation of ErbB3 (Fig. 2C) and thus confirmed the functionality of the reconstituted ErbB2/ ErbB3 complexes. Having established the Cos-7 ectopic expression model, we stained $\beta 1$-heregulin-treated cotransfected cells for nuclear ErbB3 expression. Despite the surface activation of ErbB3 receptor, we could not detect any nuclear localization (Fig. $2 \mathrm{D}$ ) or presence of the $50 \mathrm{kDa}$ fragment in the lysates (Fig. 2D), suggesting that the full-length ErbB3 receptor is not cleaved to generate a cytoplasmic fragment that translocates to the nucleus nor is it expressed in the nucleus per se.

\section{nuc-ErbB3 is an alternatively generated nuclear variant of ErbB3 RTK}

Our observations regarding the lack of cleavage of the full-length ErbB3 RTK by metalloproteases suggest the existence of an alternative mechanism that generates a nuclear variant of ErbB3. To search for putative ESTs, alternatively spliced variants, or alternative transcription initiation sites that would explain the presence of a nuclear variant of ErbB3, we used the European Molecular Biology Laboratory, the NITE Biological Research Center, and the National Center for Biotechnology Information AceView portals. AceView results can be seen following this link: http:// www.ncbi.nlm.nih.gov/IEB/Research/Acembly/av.cgi?db=human\& $\mathrm{l}=\mathrm{ERBB} 3$. The in silico analysis showed that transcription from the human ErbB3 locus produces 15 different mRNAs, 14 alter- 


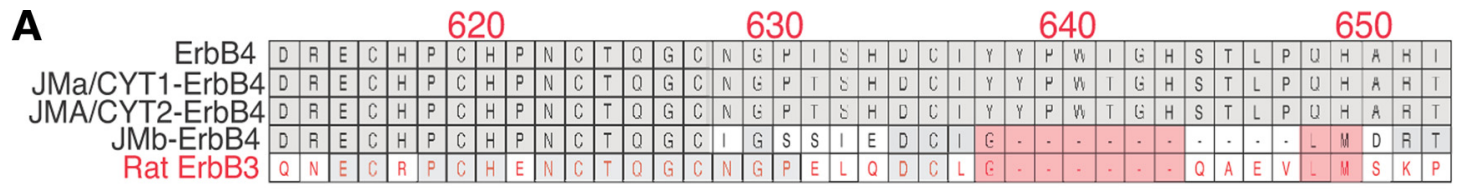

B
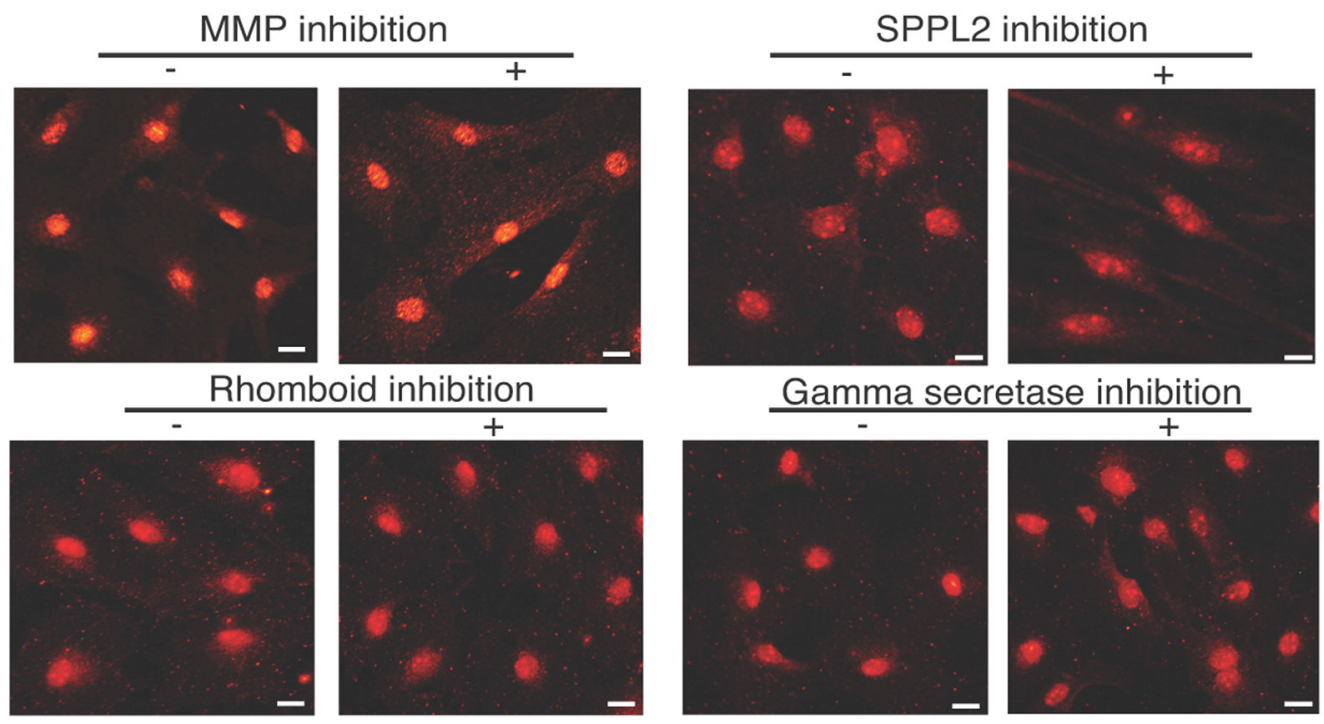

C

D

ErbB2/3 co-transfection
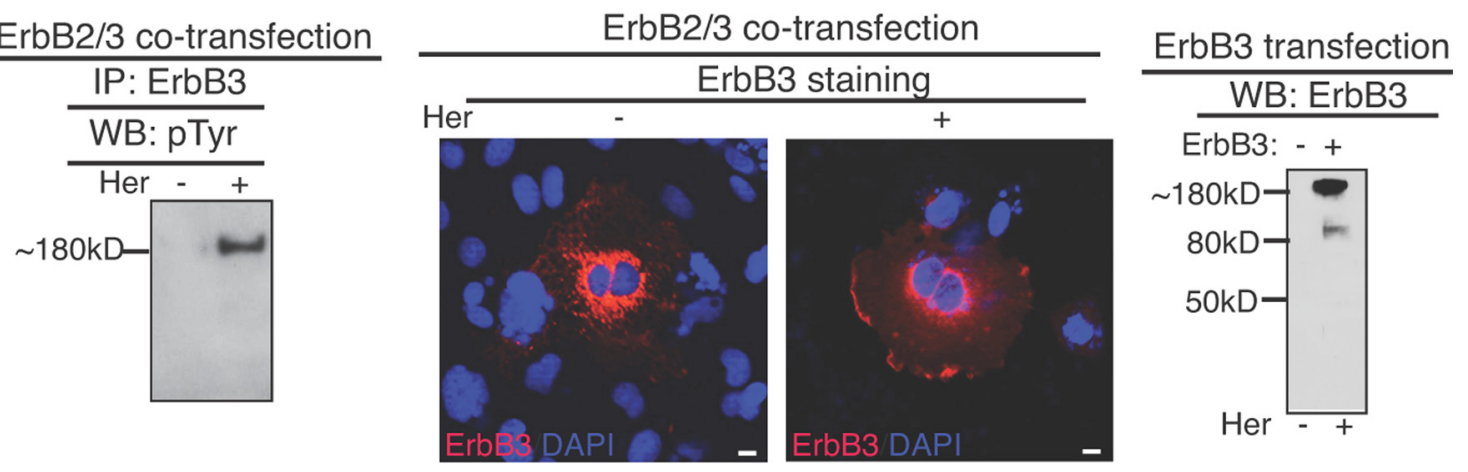

Figure 2. ErbB3 nuclear expression in rat primary Schwann cells does not depend on ligand-induced protease cleavage. $A$, Sequence comparison analysis of rat ErbB3 with various isoforms of ErbB4. ErbB3 clearly resembles the JM-b isoform that lacks the cleavage recognition site for metalloproteases. $\boldsymbol{B}$, Immunostaining for ErbB3 expression using a cytoplasmic-specific ErbB3 antibody showed no difference in nuclear ErbB3 expression after addition of $\beta 1$-heregulin in the presence of the following inhibitors: GM6001 (metalloprotease inhibitor), ZLL2 (site-2 protease), IX ( $\gamma$-secretase), DIC (Rhomboid). C, Ectopic reconstitution of functionally active ErbB2/ErbB3 heterodimers in Cos-7 cells using full-length ErbB2 and ErbB3 constructs. Addition of $\beta 1$-heregulin in cotransfected Cos-7 cells induced transphosphorylation of ErbB3, suggesting that ErbB2 and ErbB3 form active heterodimers on the cell surface. D, Cos-7 cells cotransfected with ErbB2 and ErbB3 were immunostained with ErbB3 antibody with or without the addition of $\beta 1$-heregulin. ErbB3 is localized on the membrane, in the cytoplasm, and at the perinuclear region, but not in the nucleus. Scale bars represent $20 \times$ magnification. Western blot of the cotransfected Cos-7 cells after addition of heregulin shows complete absence of the $50 \mathrm{kDa}$ band that corresponds to nuc-ErbB3.

natively spliced variants, and 1 unspliced form. There are five probable alternative promoters, six nonoverlapping alternative last exons, and three validated alternative polyadenylation sites. The mRNAs appear to differ by truncation of the $5^{\prime}$ end, truncation of the $3^{\prime}$ end, presence or absence of 26 cassette exons, overlapping exons with different boundaries, and alternative splicing or retention of 4 introns. We focused our attention to the ErbB3 variant AK125028 that was cloned mainly from human brain cDNA, includes a $5^{\prime}$-promoter and a 3 '-polyadenylation signal, and encodes a putative $50-55 \mathrm{kDa}$ protein. Cloning and expression of AK125028 in Cos-7 cells produces a $\sim 55 \mathrm{kDa}$ nuclear protein (supplemental Fig. 2, available at www.jneurosci.org as supplemental material). Translation of the AK125028 protein starts from an ATG codon located 50 nt upstream of exon 23 of the ErbB3 sequence and is part of the intron between exons 2223. The remaining of the intron upstream of the ATG is part of the 5'-UTR of AK125028. We performed a sequence comparison of AK125028 with the rat genomic ErbB3 locus to identify whether a similar ErbB3 variant exists in the genomic DNA of the rat Schwann cell. This analysis revealed that exons 23-28 are highly homologous between rat and human ErbB3, but in the human sequence the intron between exons 22-23 contains 50 unique intronic nucleotides that are part of the translated sequence in AK125028 (Fig. 3A, red box). These nucleotides are absent in the rat Schwann cell ErbB3 intron sequence and in rat ErbB3 the starting ATG codon for the putative nuclear variant is localized in the beginning of exon 23. Since in AK125028 the intron between exons $22-23$ is part of the $5^{\prime}$-UTR, we hypothesized a similar organization for the nuclear ErbB3 variant in Schwann cells. To prove this, we designed primer sets that amplify rat nuc-ErbB3 from the hypothetical 5'-UTR until exon 28 excluding all introns (Fig. 3B) and performed RT-PCR using 
B

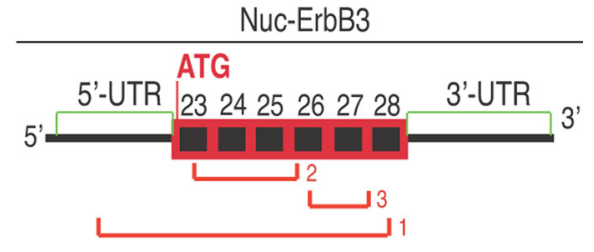

A

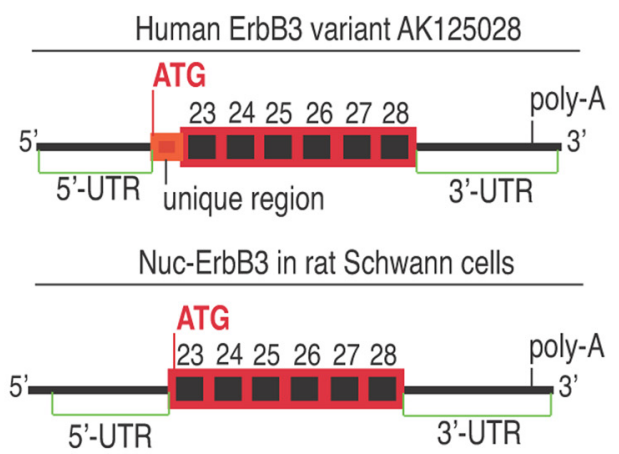

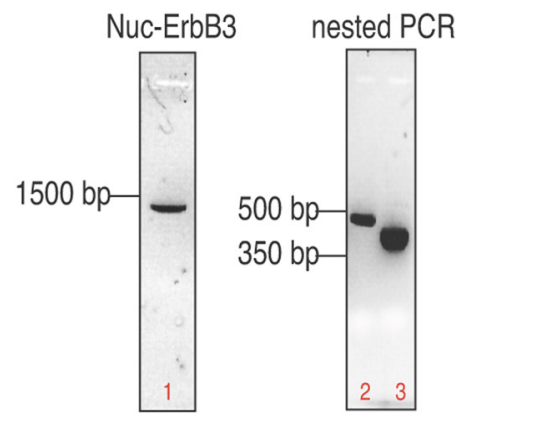

E

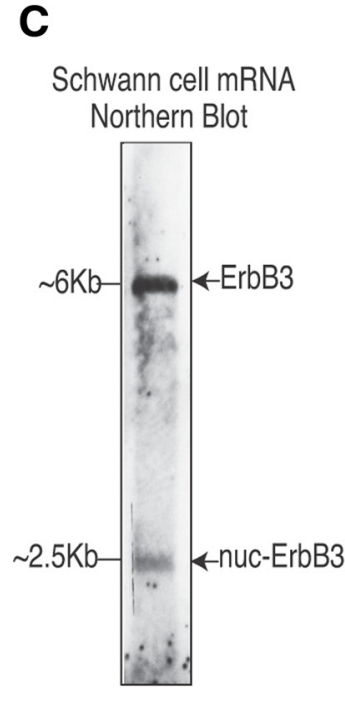

D

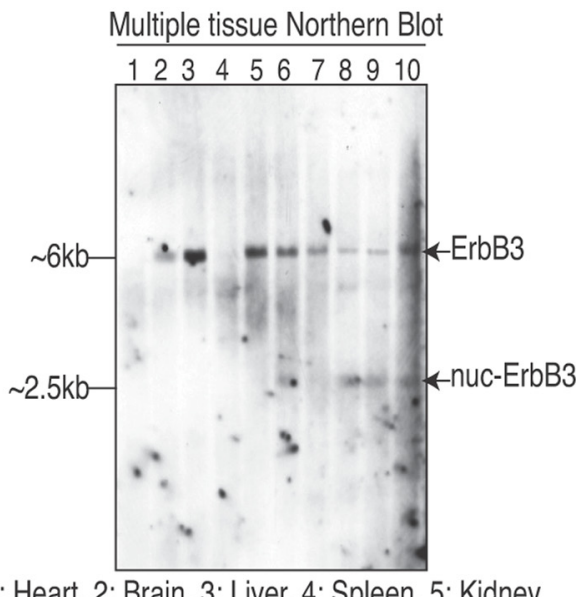

1: Heart, 2: Brain, 3: Liver, 4: Spleen, 5: Kidney 6: Embryo, 7: Lung, 8: Thymus, 9: Testes, 10: Ovary

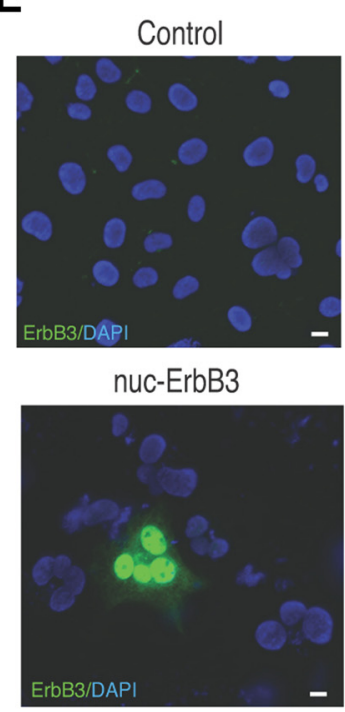

$\mathbf{F}$

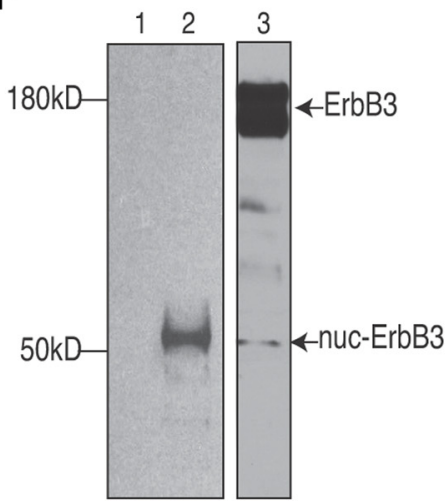

1: Cos-7 control, 2: Cos-7 + nuc-ErbB3 construct

3: Schwann cell total lysate
G

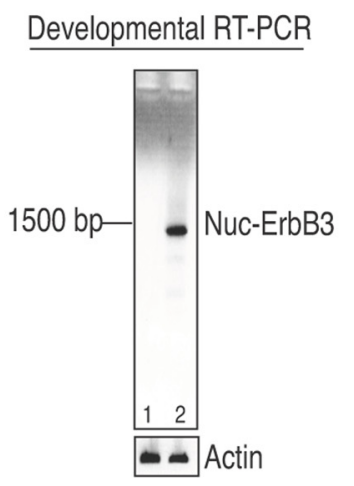

1: Adult rat sciatic nerve

2: $\mathrm{P} 2$ rat sciatic nerve

Figure 3. Identification of the nuclear variant of ErbB3 (nuc-ErbB3) in Schwann cells. A, Schematic representation of the genomic organization of the human ErbB3 nuclear variant AK125028 and the Schwann cell nuc-ErbB3 variant. Note that nuc-ErbB3 of the Schwann cell lacks the 50 nt unique region upstream of exon 23 and that its ATG codon is located at the start of exon 23 as opposed to the intronic ATG that AK125028 uses. B, Schematic showing the primer combinations used to amplify various regions of nuc-ErbB3 from Schwann cell mRNA and the respective PCR products. The nuc-ErbB3 PCR product and the nested PCR products correspond to processed mRNA excluding introns. C, Northern blotting of Schwann cell mRNA with an ErbB3 RNA probe that binds to both the full-length ErbB3 and the nuc-ErbB3 sequences reveals the existence of two ( $\sim 6$ and $\sim 2.5 \mathrm{~kb}$ ) discrete bands corresponding to the full-length ErbB3 sequence and the nuc-ErbB3, respectively. $D$, Tissue-specific expression of nuc-ErbB3. ErbB3 RNA probe cross-linked to biotin was used in Northern blotting performed on mouse mRNA from various tissues. Note that nuc-ErbB3 is expressed in whole embryos, thymus, testes, and ovaries, suggesting a broad distribution of expression. E, Transfection of Cos-7 cells that lack endogenous ErbB3 with nuc-ErbB3 construct showed that nuc-ErbB3 is localized to the nucleus. Control represents vector-transfected cells stained for ErbB3 expression. F, Western blotting on Cos-7 cell lysates transfected with the Schwann cell-derived nuc-ErbB3 construct reveals a $50 \mathrm{kDa}$ protein that migrates equally with the endogenous Schwann cell nuc-ErbB3 band. G,Developmental profile of nuc-ErbB3 expression by RT-PCR using RNA from adult rat (Figure legend continues.) 
oligo-dT cDNA from Schwann cell mRNA. We also designed primers that target exonic regions only and performed nested PCR using the purified nuc-ErbB3 PCR product as a template (Fig. 3B). The data presented in Figure $3 B$ represent processed mRNA (excluding introns) and not pre-mRNA. The identity of the amplified fragments was confirmed by restriction digest and sequencing. These experiments proved the presence of a new ErbB3 variant (nuc-ErbB3) in the pool of Schwann cell mRNAs with an independent 5 '-UTR from parts of the intron between exons 22 and 23 (Fig. 3B). To further confirm the presence of nuc-ErbB3 in the mRNA of the Schwann cell, we designed a RNA probe covering exons 23-27 and performed Northern blotting using rat primary Schwann cell mRNA. This revealed the existence of two discrete bands, one at $\sim 6 \mathrm{~kb}$ that correlates to the full-length ErbB3 sequence and one at $\sim 2.5 \mathrm{~kb}$, which correlates with the size of the nuc-ErbB3 sequence including the nucleotides of the 5'- and 3'-UTRs (Fig. 3C). Additionally, we used a multiple tissue Northern blot loaded with mRNA from various mouse tissues (Ambion) to examine the presence of nuc-ErbB3. nuc-ErbB3 transcript was present in mRNA isolated from whole embryos, thymus, testes, and ovaries, suggesting a broad distribution of expression (Fig. 3D). Finally, we cloned and expressed Schwann cell's nuc-ErbB3 in Cos-7 cells using the pcDNA3.1/His mammalian expression vector (Invitrogen). Immunofluorescence detection using an ErbB3 C-terminal specific antibody showed that nuc-ErbB3 is expressed in the nucleus of Cos-7 cells (Fig. 3E). Western blotting on Cos-7 cell lysates after transfection with nuc-ErbB3/His showed that nuc-ErbB3 protein runs at $\sim 50$ $\mathrm{kDa}$ at the same size as the endogenous ErbB3-reacting band in Schwann cell nuclear extracts (Fig. 3F). We used Cos-7 cells for these experiments because they do not express endogenous ErbB3 so it is easier to interpret the expression data. In conclusion, these results confirmed the existence of the nuc-ErbB3 variant in Schwann cells as a mature mRNA capable of mediating translation of a nuclear protein. The sequence of the nuc-ErbB3 of the Schwann cell has been deposited to the GenBank under the accession number GU598254.

\section{In vivo developmental profile of nuc-ErbB3 expression}

To determine the developmental profile of nuc-ErbB3 in vivo, we isolated total RNA from adult rat sciatic nerves and from P2 rat sciatic nerves. We performed RT-PCR using the primer set that specifically detects nuc-ErbB3 and showed that is expressed in sciatic nerves of $\mathrm{P} 2$ rat pups but not in sciatic nerves of adult rats (Fig. 3G). This suggests that nuc-ErbB3 expression coincides with the mitogenic/migratory phase of Schwann cells, whereas as Schwann cells terminally differentiate and the myelination program matures, nuc-ErbB3 expression is abrogated. The developmental profile of nuc-ErbB3 suggests that the presence of nucErbB3 in peripheral nerves may depend on the threshold of neuregulin signaling, which is the main mitogenic signal for Schwann cells, a hypothesis that is supported by our results regarding the regulation of nuc-ErbB3 translation by neuregulin in Schwann cells (see Fig. 5A).

\section{nuc-ErbB3 contains an active NLS motif}

Next, we sought to identify how nuc-ErbB3 is localized to the nucleus of Schwann cells. Eukaryotic proteins are usually trans-

\section{$\leftarrow$}

(Figure legend continued.) sciatic nerves and P2 rat sciatic nerves shows that nuc-ErbB3 depends on the differentiation state of Schwann cells and is abrogated in adult myelinated nerves in vivo. Actin was used as a housekeeping gene to normalize expression. ported into the nucleus either through passive transport [efficient for small-size proteins (Sweet and Gerace, 1995)] or by means of active transportation using the cellular nucleo-cytoplasmic shuttling machinery at the expense of cellular energy (Bednenko et al., 2003). Proteins that are capable of being actively transported into the nuclear complex bear distinct NLS sequences (Xu and Massague, 2004). To identify a putative NLS sequence in nuc-ErbB3, we performed an extensive motif search through PredictNLS, DBS-PRED, and SubLoc, version 1.0, servers. The consensus topology of nuc-ErbB3 was predicted as nuclear. In addition, the existence of the NLS sequence RSRSRSPRPR was predicted between amino acids 296 and 305 of nuc-ErbB3 (that correspond to amino acids 1118-1127 of the full-length ErbB3 sequence) (Fig. $4 A)$. Several mammalian proteins carry this particular NLS sequence, and all are nuclear proteins (supplemental Table 1, available at www.jneurosci.org as supplemental material). To test the functionality of this NLS, we performed site-directed mutagenesis to substitute the third arginine in the NLS recognition motif to alanine in nuc-ErbB3. Heterologous expression in Cos-7 cells revealed that this isoform fails to translocate to the nucleus, suggesting that the R300A mutation is sufficient to abrogate the nuclear localization (Fig. $4 B$ ) and that the predicted NLS is functional.

\section{ChIP-chip array reveals that nuc-ErbB3 associates with Schwann cell promoters}

To determine whether nuc-ErbB3 associates with DNA in Schwann cells, we performed a genome-wide ChIP-chip array analysis using the array design of NimbleGen. After normalization and background extraction, the ChIP-chip array analysis revealed 597 significant peaks lying within promoter regions across the entire rat genome. In addition, we performed a gene expression analysis in Schwann cells using Affymetrix gene chips. Finally, genes that are present in Schwann cells and contain promoters that interact with nuc-ErbB3 were identified from the mRNA expression patterns and ChIP on ChIP data, respectively, in the following way. Gene accession numbers or gene descriptions were obtained from the reports of Affymetrix expression results and NimbleGen ChIP on ChIP experiments. Corresponding protein sequences were extracted from GenBank using an in-house script. A total of 6887 genes was identified for the set characterizing Schwann cell expression (reference set), and 589 genes were identified with promoters that interact with nucErbB3 (activation set). Genes were grouped using a greedy incremental clustering algorithm implemented in the program CD-HIT (Li et al., 2001) at $90 \%$ sequence identity. Clustering results did not change when cutoff values were varied in the range of $80-95 \%$ sequence identity. There was some redundancy in the datasets, which reduced the reference set to 6488 genes and the activation set to 478 genes with promoters that interact with nucErbB3. When these two nonredundant sets were compared with each other using CD-HIT, 63 genes were identified that were present in both the reference and activation sets. This means that, from the 478 promoters that nuc-ErbB 3 binds, a total of 63 genes with promoters that interact with nuc-ErbB3 is expressed in Schwann cells. The accession numbers and descriptions of these 63 genes are included in the supplemental material (supplemental Table 2, available at www.jneurosci.org as supplemental material). These genes correspond to $\sim 1.2 \%$ of the total expressed genes in Schwann cells and $\sim 10 \%$ of the genes that interact with nuc-ErbB3. To functionally annotate these genes we used the Database for Annotation, Visualization, and Integrated Discovery (DAVID) (Dennis et al., 2003), which revealed nine statistically significant functional clusters $(p<0.005)$ (Fig. $4 C)$. The 


\section{A nuc-ErbB3 NLS: 296 - R S R S R S P R P R - 305 \\ nuc-ErbB3 mut-NLS: 296 - R S R S A S P R P R - 305}

\section{B}

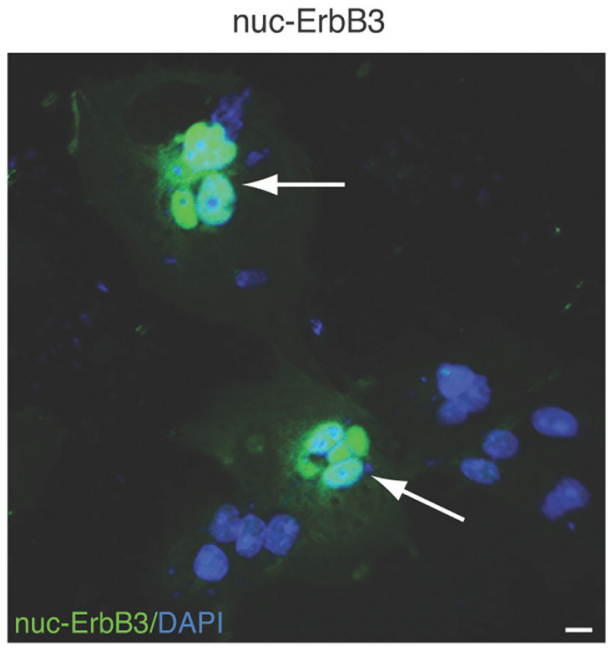

nuc-ErbB3 mut-NLS

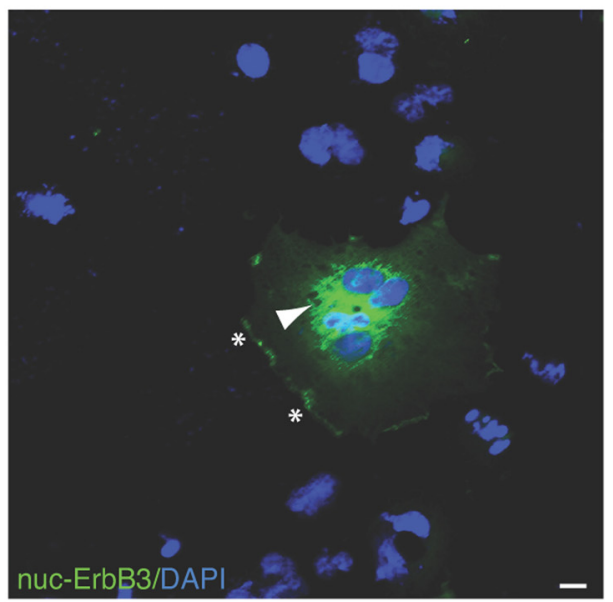

C

Functional Clusters

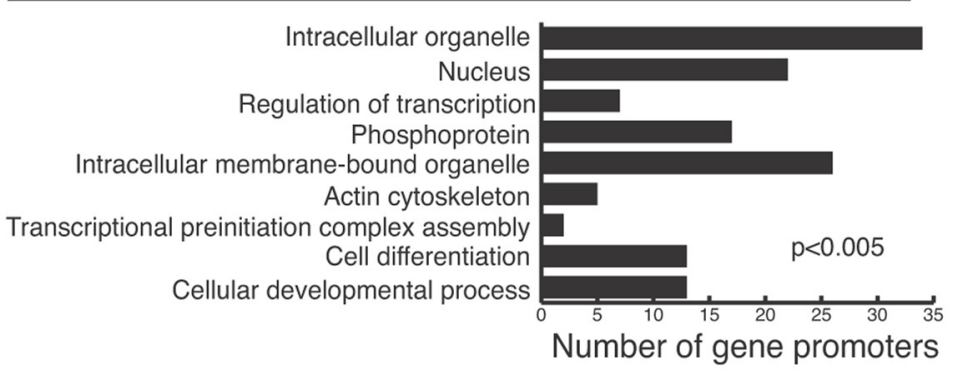

\section{D}
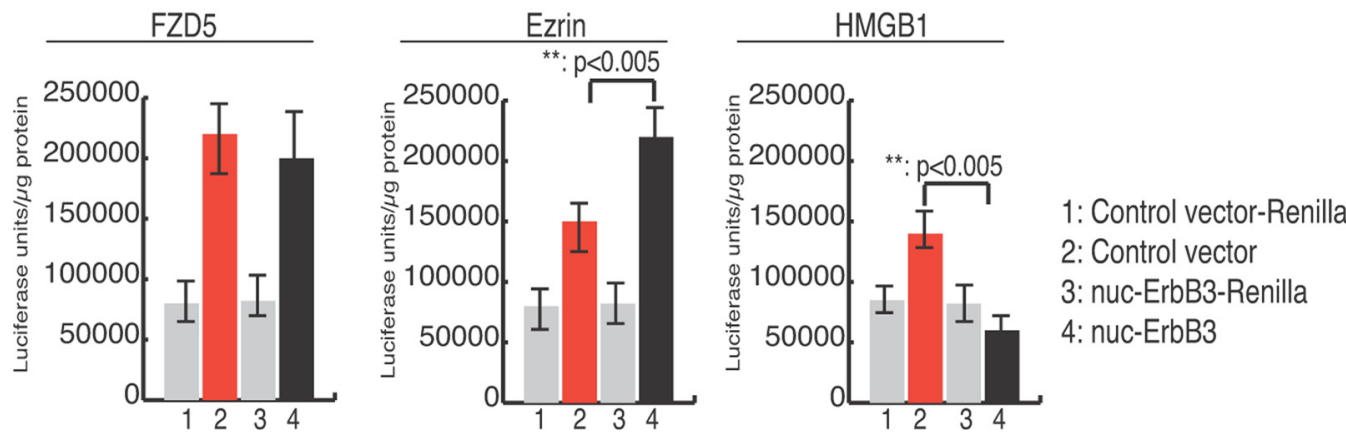

Figure 4. nuc-ErbB3 translocates to the nucleus because of an active NLS sequence. $A$, Computational analysis of the nuc-ErbB3 sequence revealed a classical NLS sequence between amino acids 296 and 305. B, nuc-ErbB3 variant from rat primary Schwann cells was cloned into pCDNA 3.1/TOPO and the NLS sequence was mutated using QuikChange mutagenesis kit (Stratagene) by substituting arginine at position 300 with alanine (R300A). Both wild-type and the mutant plasmids were transfected into Cos-7 cells and stained with C-ErbB3 antibody $72 \mathrm{~h}$ after transfection. This showed that point mutation R300A abolishes nuclear localization. The arrows indicate nuclear staining; arrowheads, perinuclear staining; and asterisks, membranous staining. C, nuc-ErbB3 associates with a range of gene promoters as revealed by genome-wide ChIP-chip array. In $S$ chwann cells, nuc-ErbB3 associates with genomic regions that contain promoters of 63 expressed genes. Functional clustering of these 63 Schwann cell genes using DAVID reveals nine significant functional clusters $(p<0.005)$. D, nuc-ErbB3 directly regulates the promoter activities of ezrin and HMGB1 genes $(p<0.005)$ but not Frizzled-5 promoter as quantified by dual luciferase assays. The graphs represent the average of six independent experiments, and the luciferase units have been normalized to DNA content, protein content, and the expression of Renilla luciferase.

functional significance of the clustered genes in central biosynthetic, biochemical, and transcriptional pathways of Schwann cells suggest an important role of nuc-ErbB3 in gene regulation.

\section{nuc-ErbB3 regulates the promoter activity of Schwann cell genes}

To identify whether nuc-ErbB3 has a transcription regulatory activity, we performed luciferase assays using a nuc-ErbB3- expressing construct and promoter/luciferase constructs (purchased from SwitchGear Genomics) that correspond to the 63 Schwann cell genes (see our ChIP-chip data). We cotransfected nuc-ErbB3 with the promoter/luciferase constructs in Cos-7 cells that do not express endogenous ErbB3 nor nuc-ErbB3 and quantified the amount of luciferase compared with control cells, which were cotransfected with the promoter/luciferase construct and an empty vector in equimolar concentration with nuc-ErbB3. The 
results were normalized to protein content and against the expression of Renilla luciferase to neutralize differences in transfection efficiency. The data presented here correspond to the regulation of Ezrin, Frizzled-5, and HMGB1 promoter activities by nuc-ErbB3. The selection of Frizzled-5 and HMGB1 promoters was based on construct availability, and even though their role in Schwann cell development and myelination is unclear we were interested to see whether nuc-ErbB3 regulates their expression since they appear in the list of nuc-ErbB3-associated promoters (supplemental Table 2, available at www.jneurosci.org as supplemental material). nuc-ErbB3 significantly $(p<0.005)$ enhances the transcriptional activity of ezrin promoter, does not directly regulate the promoter activity of Frizzled-5, and significantly represses the promoter activity of HMGB1 transcription factor (Fig. 4D). These results suggest that nuc-ErbB3 functions as a transcriptional regulator in Schwann cells. Luciferase assays with the remaining 60 promoter constructs are currently undergoing.

\section{Heregulin regulates the translation of nuc-ErbB3}

In mammalian cells, the expression of protein-encoding genes requires a series of steps in which pre-mRNA is processed to $\mathrm{mRNA}$ in the nucleus before mRNA is translated into protein in the cytoplasm. These steps are subject to quality control to ensure that only completely processed mRNA is exported to the cytoplasm (Maquat and Carmichael, 2001). An additional quality control, called nonsense-mediated mRNA decay (NMD), degrade mRNAs that prematurely terminate translation $>50-55 \mathrm{nt}$ upstream of an exon-exon junction as a means to prevent the synthesis of potentially harmful truncated proteins (Maquat, 1995). The cap binding protein 80 (CBP80) shuttles in association with mRNA to the cytoplasm and the CBP80-bound mRNA is the primary template for the first so-called "pioneer" round of translation since it is the primary substrate of NMD (Ishigaki et al., 2001). After this quality check, eIF4E replaces CBP80 and the mRNA is subject to steady-state translation (Ishigaki et al., 2001). To identify whether neuregulin signaling regulates the translation of nuc-ErbB3 and whether nuc-ErbB3 mRNA is translated into a mature protein in Schwann cells, we performed RT-PCR on mRNA that is immunoprecipitated in complex with eIF4E in the presence or absence of neuregulin. We identified that neuregulin regulates the steady-state translation of both the fulllength ErbB3 receptor and the nuc-ErbB3 in Schwann cells (Fig. $5 A$ ) since both mRNAs are bound to eIF4E in the cytoplasm of Schwann cells and this association increases in the presence of neuregulin. To examine whether the effect of neuregulin on nucErbB3 translation is specific, we performed an RT-PCR on eiF4Eprecipitated mRNA for $\beta$-actin as a housekeeping gene. This showed that neuregulin does not affect the translation of actin (Fig. 5A) mRNA. However, we cannot exclude that the effect of neuregulin on translation is a result of the general mitogenic/ trophic role of neuregulin for Schwann cells and does not reflect a specific regulation of ErbB3 mRNA. In either case, our data show that the rate of translation of nuc-ErbB3 is directly regulated by the presence of neuregulin in Schwann cells.

\section{nuc-ErbB3 regulates myelination in Schwann cell-neuron cocultures}

To examine the role of nuc-ErbB3 during the interaction of Schwann cells with axons, we designed siRNAs that selectively target nuc-ErbB3 expression without affecting the expression of the full-length ErbB3 receptor and subsequently the neuregulinErbB3 signaling axis. To achieve this, we designed siRNAs that target the $5^{\prime}$-UTR of the nuc-ErbB3 variant (see Fig. $3 A$ for the organization of nuc-ErbB3 gene and GenBank accession number GU598254). We transfected the nuc-ErbB3 siRNA and a scrambled nuc-ErbB3 control siRNA into rat primary Schwann cells using nucleofection and examined the knockdown efficiency by Western blotting. To verify the specificity of the knockdown, we performed a concentration gradient of the siRNAs between 20 and $400 \mathrm{~nm}$ (data not shown). We decided to use $400 \mathrm{~nm}$ for all our experiments since this concentration provided the best knockdown efficiency with no apparent side targeting. These experiments showed that nuc-ErbB3 siRNA does not affect the expression of the full-length ErbB3 receptor (Fig. 5B, cytoplasmic extracts; $C$, left graph), whereas it inhibits the expression of nucErbB3 by $\sim 30-40 \%$ (Fig. $5 B$, nuclear extracts; $C$, right graph) compared with the scrambled control siRNA expression. We obtained similar results using a second set of siRNAs, which targets the $\mathrm{N}^{\prime}$-terminus of ErbB3 between nucleotides 263-288 (fulllength ErbB3-siRNA) and the cytoplasmic domain of ErbB3 that targets both forms (full-length ErbB3 and nuc-ErbB3) (supplemental Fig. 3, available at www.jneurosci.org as supplemental material).

Since the neuregulin/ErbB3 signaling axis has been previously associated with the regulation of Schwann cell proliferation (Dong et al., 1995; Levi et al., 1995; Morrissey et al., 1995), maturation (Dong et al., 1995), and survival (Syroid et al., 1996), we examined the effects of the nuc-ErbB3-siRNA on these processes. Quantification of BrdU-positive Schwann cells expressed as percentage of the total number of Schwann cells in culture showed that the nuc-ErbB3 siRNA has no effect on the proliferation rate of Schwann cells (Fig. 5D). Finally, quantification of the percentage of caspase-positive cells (marker of cell apoptosis) compared with the total number of Schwann cells in culture showed that the nuc-ErbB3 siRNA does not affect the apoptotic rate of Schwann cells (Fig. 5E).

To reveal the putative function of nuc-ErbB3 on Schwann cell-neuron interactions and myelination, we prepared cocultures of purified DRG neurons and siRNA-transfected Schwann cells. Seventy-two hours after the addition of Schwann cells to DRG axons, we treated the cocultures with ascorbic acid to initiate myelination as previously described (Windebank et al., 1985; Carey and Todd, 1987). Fourteen days after the induction of myelination, we observed a significant reduction of the amount of myelinated segments in the nuc-ErbB3 siRNA-transfected cultures compared with scrambled nuc-ErbB3 siRNA or control cultures (Fig. $6 A, B$ ). The number of Schwann cells present in the cocultures was equal at the time of myelin quantification as measured by the total number of DAPI-positive cells in the cultures (Fig. 6C), which together with the fact that the siRNAs have no effect on Schwann cell proliferation or death (Fig. 5D,E) implies that the difference in myelination can be attributed directly to nuc-ErbB3 function. The decrease in myelination after inhibition of nuc-ErbB3 expression, although significant ( $p<0.05$ ), is subtle compared with the total loss of myelination after inhibition of the full-length ErbB3. This suggests that the proper expression of nuc-ErbB3 in Schwann cells influences the extent of Schwann cell myelination in a way that complements the major role of the full-length ErbB3 receptor tyrosine kinase.

\section{nuc-ErbB3 affects the distribution of ezrin at the nodes of Ranvier}

Ezrin, radixin, and moesin, also known as ERM proteins, are members of the band 4.1 superfamily of proteins and are expressed differentially in the microvilli of many cell types (Berryman et al., 1993). Recently, it was shown that ezrin is a component of 
A
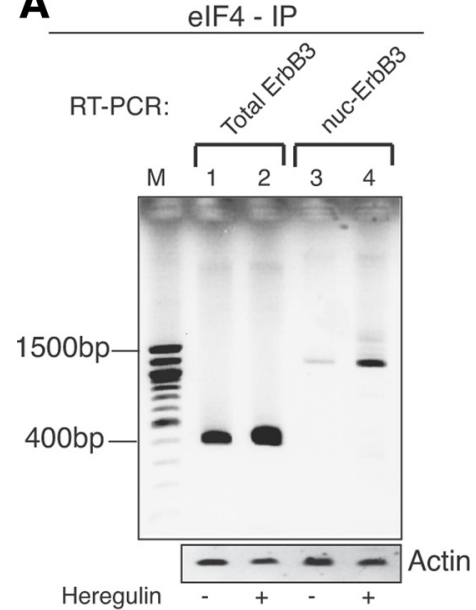

1,3: elF4-IP

2,4: elF4-IP + Heregulin

C

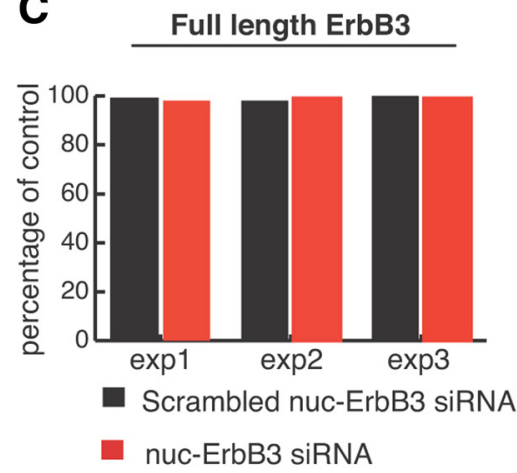

D

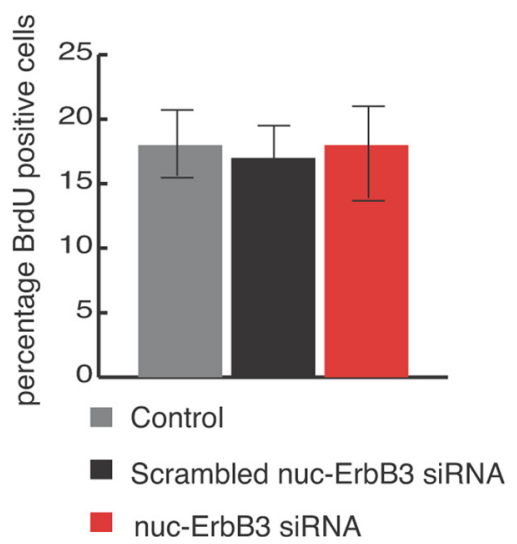

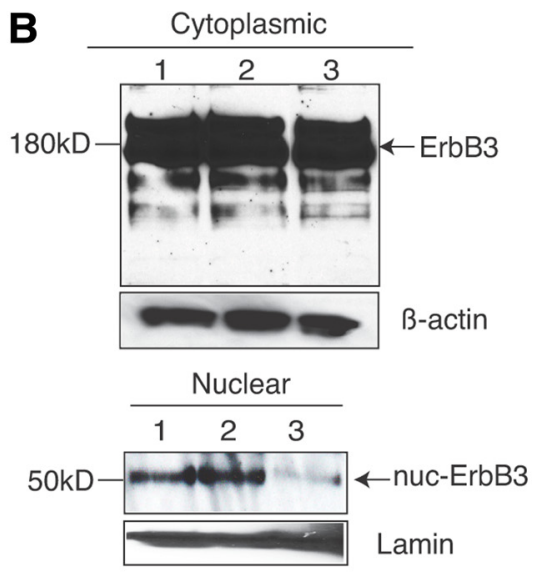

\section{1: Control \\ 2: Scrambled nuc-ErbB3 siRNA 3: nuc-ErbB3 siRNA}

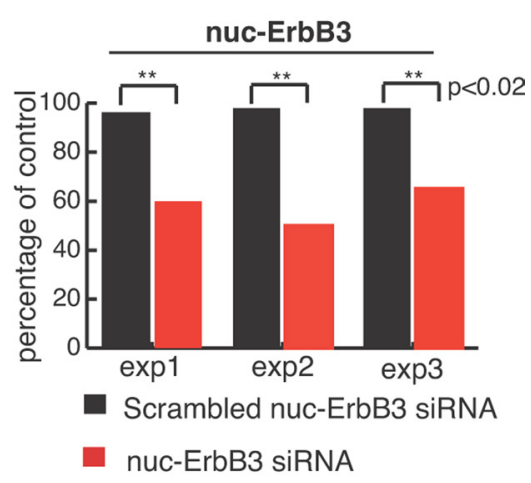

E

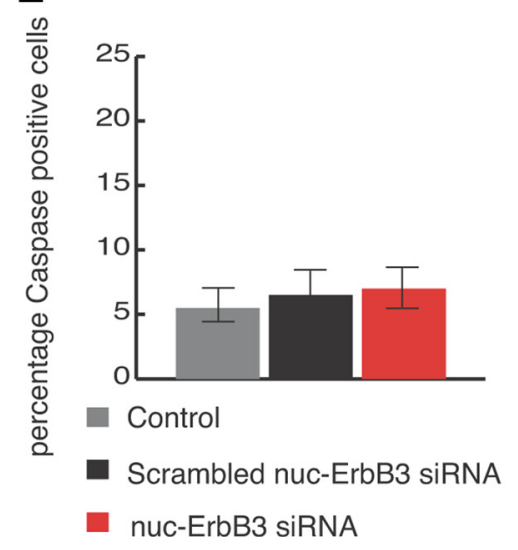

Figure 5. Translation of nuc-ErbB3 is regulated by neuregulin and siRNAs can inhibit nuc-ErbB3 expression in Schwann cells. $A$, Neuregulin increases the steady-state translation rate of nuc-ErbB3 and full-length ErbB3 in Schwann cells as shown by RT-PCR on elF4E-bound mRNA. Actin RT-PCR on eif4E precipitated mRNA was performed to determine the specificity of the neuregulin effect on nuc-ErbB3 translation. $\boldsymbol{B}$, Transfection of nuc-ErbB3 siRNA into Schwann cells downregulates the expression of nuc-ErbB3 in nuclear extracts compared with control-siRNA (scrambled nuc-ErbB3 siRNA)-transfected cells but does not affect the expression of full-length ErbB3 in the cytoplasmic/membrane extracts. Actin and lamin A/C were used as loading controls for cytoplasmic and nuclear extracts, respectively. C, Densitometric analysis from three independent experiments shows that the nuc-ErbB3 siRNA does not affect the expression of full-length ErbB3 but induce a significant inhibition of nuc-ErbB3 expression $\left({ }^{* *} p<0.02\right)$ as quantified using a paired $t$ test. The graphs are normalized to the average expression of full-length ErbB3 or nuc-ErbB3 in Schwann cell cultures treated with scrambled nuc-ErbB3 siRNAs. D, The nuc-ErbB3 siRNA does not affect the proliferation rate of Schwann cells as quantified by incorporation of BrdU. The graph shows the average of BrdU-positive cells as a percentage of DAPI-positive cells in the culture from three independent coverslips. $\boldsymbol{E}$, Transfection of Schwann cells with nuc-ErbB3 siRNA does not alter their apoptotic rate. Apoptosis was quantified as the percentage of caspase-positive cells in the culture from three coverslips.

Schwann cell microvilli (Scherer et al., 2001) and that nodes of Ranvier in the peripheral nervous system (PNS) are formed in association with ERM-positive Schwann cell processes (Melendez-Vasquez et al., 2001). Ezrin is tightly clustered in the nodes of mature myelinated fibers and ezrin clustering precedes compact myelin formation, whereas as myelinating Schwann cells mature, ezrin clusters become tighter at the nodal region (Melendez-Vasquez et al., 2001). Since nuc-ErbB3 activates the transcriptional activity of ezrin promoter (Fig. $4 D$ ), we examined the effect of the inhibition of nuc-ErbB3 expression on the distribution of ezrin in Schwann cell processes that outline the nodes of Ranvier. We transfected Schwann cells with the nuc-ErbB3 siRNA and the scrambled control-siRNA and then plated them on purified DRG axons to induce myelination. Fourteen days after the induction of myelination with ascorbic acid, we stained the cocultures for MBP and ezrin expression. In the control siRNA cultures, ezrin was primarily distributed tightly at the nodes (Fig. 7Aa, arrow; $B$ ), which is in agreement with previous reports showing ezrin expression at the nodes in adult myelinated fibers (MelendezVasquez et al., 2001). In the nuc-ErbB3 siRNA-transfected cultures, the number of ezrin-positive nodes (Fig. 7Ab, arrow) was significantly decreased (Fig. $7 B$ ), whereas the expression of ezrin in apposed heminodes (Fig. $7 A b, c$, asterisk) or in single heminodes (Figs. $7 A b, d$, arrowhead) was significantly increased $(p<0.05)$ compared with the control siRNA-transfected cultures (Fig. 7B). The expression of ezrin in heminodes and the reduction of tight expression in the nodes (Fig. $7 B$ ) suggest a delay in maturation of the myelinating Schwann cells in the culture. This pattern of expression resembles early developmental stages of the PNS (Melendez-Vasquez et al., 2001) and implies that nuc-ErbB3 regulates the proper timing of ezrin clustering in the Schwann cell processes that surround the nodes of Ranvier. This may either be a direct regulation of ezrin expression through the binding and transcriptional activation of the ezrin promoter (Fig. 4) or may be indirect since inhibition of nuc-ErbB3 expression results in reduced rate of myelination (Fig. 6) probably through a complex network of signaling and transcriptional interactions.

\section{Discussion}

Reciprocal interactions between glia and neurons are essential for the proper organization and function of the nervous system. In this context, the importance of the NRG1-ErbB2/3 signaling axis is indisput- 
A
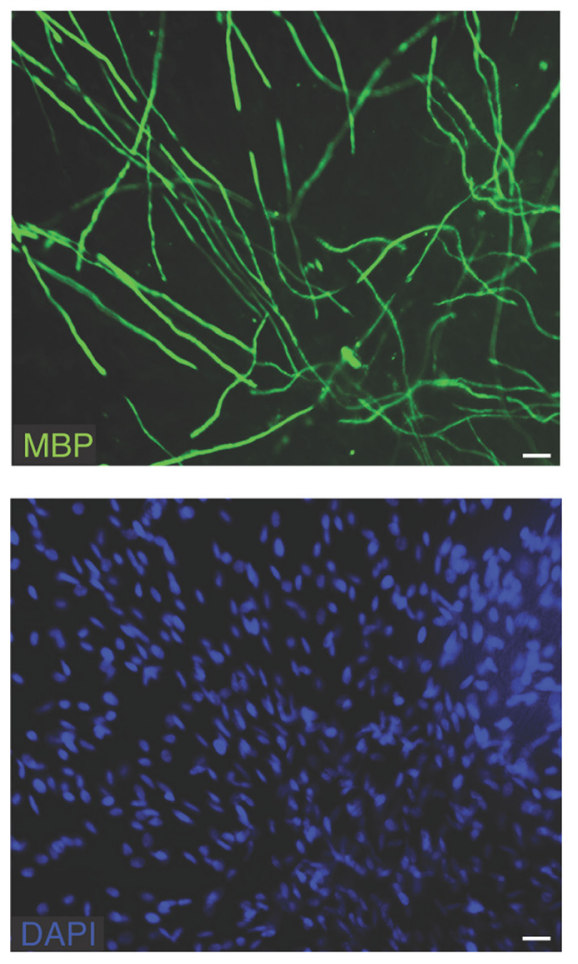

B

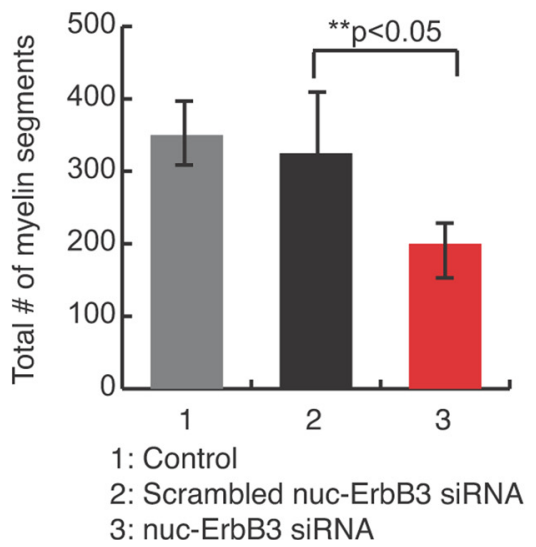

Scrambled nuc-ErbB3 siRNA
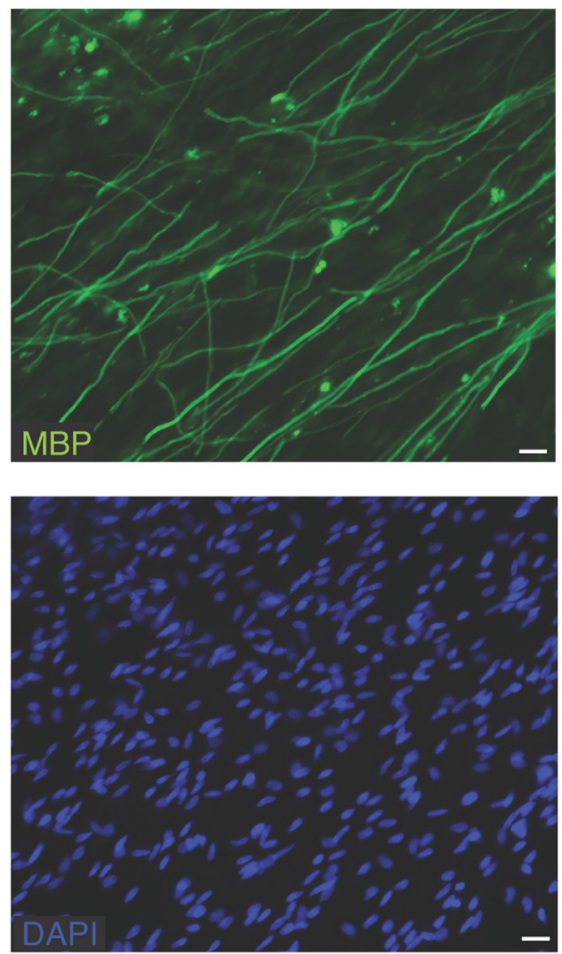

nuc-ErbB3 siRNA
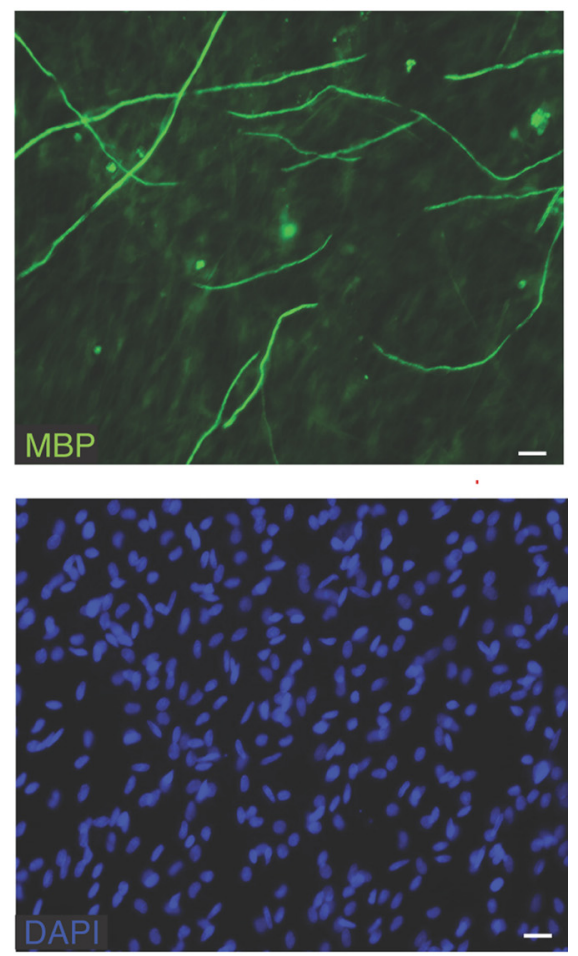

C

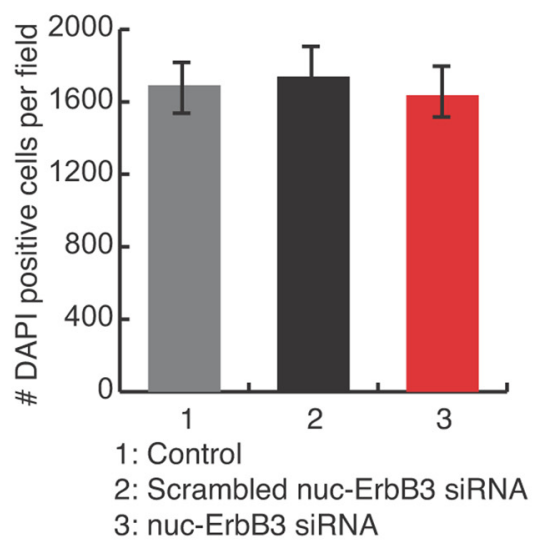

Figure 6. nuc-ErbB3 influences Schwann cell myelination. $A$, nuc-ErbB3 siRNA-transfected Schwann cell neuron cocultures produce significantly less compact myelin compared with scrambled nuc-ErbB3 siRNA-transfected cultures or control (nontransfected) cultures. Myelin is stained with anti-MBP antibodies (green), and DAPI shows the amount of cells in the field. $\boldsymbol{B}$, Quantification of the number of myelin segments from three independent experiments $(n=10)$ shows that the reduction in the number of myelinated segments in nuc-Erbb3 siRNA-transfected cultures is significant $(p<0.05)$. C, Quantification of the number of DAPI-positive cells per field of the myelinated Schwann cell neuron cocultures shows that the amount of cells is equal between the control and the siRNA-transfected cultures.

able; however, questions remain regarding the exact role of the axis in the PNS, such as how NRG1 signaling drives proliferation and/or differentiation of Schwann cells and how NRG1 signaling strength regulates the binary choice of Schwann cell phenotypes. To address some of these questions, we are studying the functional role of ErbB3 RTK since it is the specific receptor for NRG1 on the surface of Schwann cells. Here, we report the existence of a nuclear variant of ErbB3 encoding a $50 \mathrm{kDa}$ protein (nuc-ErbB3), which has a distinct role in the regulation of Schwann cell myelination.

Although nuclear localization of ErbB3 has been already reported in Schwann cells (Raabe et al., 2004), the mechanism for generating nuclear ErbB3 and its functional significance remained unknown. Recently, both biochemical (Vecchi and Car- penter, 1997; Ni et al., 2001; Lee et al., 2002) and molecular (Muraoka-Cook et al., 2006; Sardi et al., 2006) studies have shown cleavage of ErbB4 by $\gamma$-secretase and translocation of the cleaved ErbB4 cytoplasmic domain in the nucleus of mammary epithelial cells (Muraoka-Cook et al., 2006) and astrocytes (Sardi et al., 2006). We examined the possibility that ErbB3 is cleaved by regulated intramembrane proteolysis and the cytoplasmic domain translocates to the nucleus of Schwann cells, which abides by the model established for Notch (Steiner and Haass, 2000) and ErbB4 (Carpenter, 2003). Our data show that inhibition of metalloproteases or endoproteases like $\gamma$-secretase, SPPL-2, and Rhomboid in Schwann cells does not affect the expression of ErbB3 in the nucleus. Similarity of ErbB3 to noncleavable isoform (JM-b) of ErBB4 with respect to the lack of a metallopro- 
A
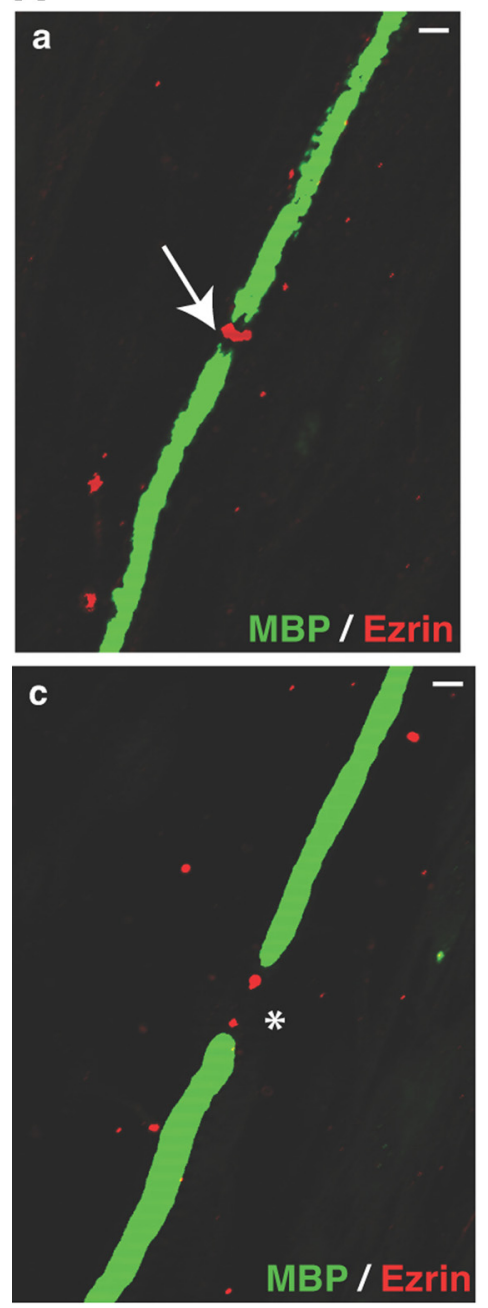
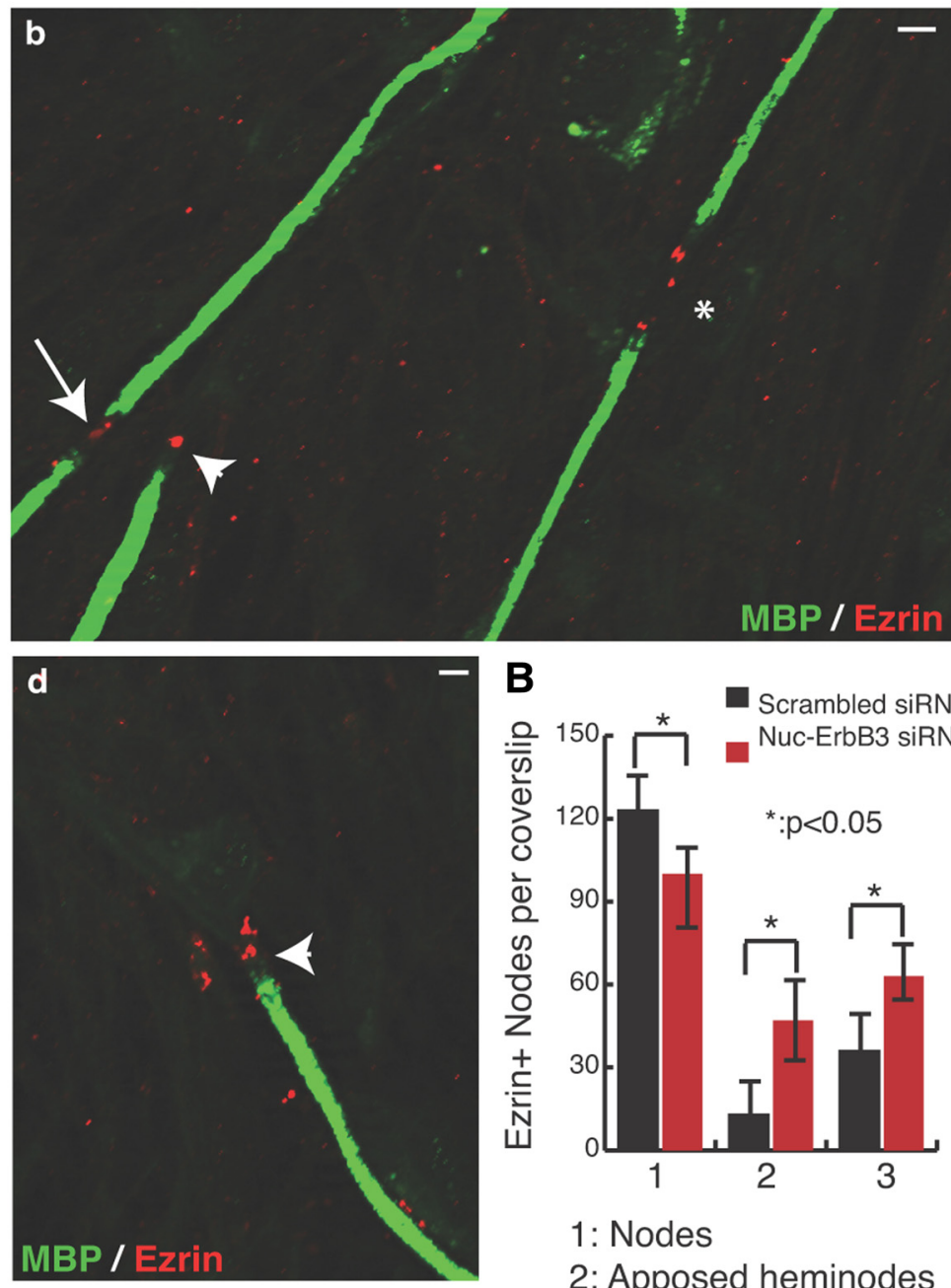

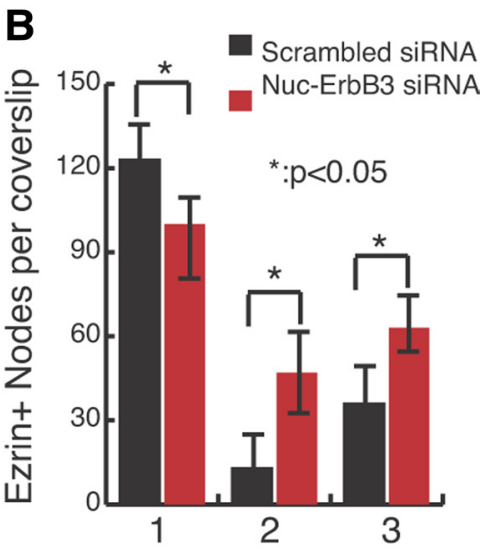

1: Nodes

2: Apposed heminodes

3: Single heminodes

Figure 7. Inhibition of nuc-ErbB3 expression affects the distribution of ezrin at the nodes of Ranvier. $\boldsymbol{A}$, In scrambled nuc-ErbB3 siRNA-transfected cocultures, ezrin is expressed tightly at the nodal region ( $\boldsymbol{a}$, arrow). In nuc-ErbB3 siRNA cultures, ezrin is expressed at the nodes ( $\boldsymbol{b}$, arrow), in apposed heminodes ( $\boldsymbol{b}, \boldsymbol{c}$, asterisk), and in single heminodes ( $\boldsymbol{b}, \boldsymbol{d}$, arrowheads). $\boldsymbol{B}$, Quantification of the number of ezrin-positive nodes reveals a significant $\left({ }^{*} p<0.05\right)$ reduction of ezrin expression in tight nodes and an increase in apposed heminodes and single heminodes in nuc-ErbB3 siRNA-transfected cultures compared with scrambled nuc-ErbB3 siRNA-transfected cultures. The graph shows the average number of ezrin-positive nodes per coverslip \pm SD. Significance was calculated using a paired Student $t$ test from a total of six coverslips per condition.

tease recognition motif (Fig. 2A) further confirms that nucErbB3 is generated by a different mechanism.

Alternative splicing and differential promoter usage is documented in the case of NRG1 giving rise to at least 15 different isoforms that demonstrate different spatiotemporal patterns of expression (Meyer et al., 1997). Alternate transcriptional splice variants encoding different isoforms have been characterized for ErbB3. One isoform encoded by a $2.1 \mathrm{~kb}$ transcript was detected by a $\mathrm{N}$-terminal-specific probe, lacks the intermembrane region, and is secreted outside the cell (Chen et al., 2007). This form acts to modulate the activity of the membrane-bound form. Additional variants have also been reported for human ErbB3 (National Institutes of Health AceView portal), some of which putatively encode proteins, suggesting that alternative splicing or alternative transcription initiation are indeed used for regulating ErbB3 function. Heterologous expression of one specific isoform predicted for human ErbB3 (AK125028) in Cos-7 cells encoded a $\sim 50 \mathrm{kDa}$ protein that localized in the nucleus (supplemental data, available at www.jneurosci.org as supplemental material).
Here, we show the existence of a novel ErbB3 variant (nucErbB3) in the Schwann cell mRNA pool consisting of exons 23-28 of the full-length ErbB3 gene with an independent 5' -UTR (Fig. 3). nuc-ErbB3 in Schwann cells shares the same gene architecture with the human AK125028 variant (Fig. 3). The fact that nuc-ErbB3 has a functional ATG codon, an independent $5^{\prime}$ UTR, and that the first exon of the parental ErbB3 gene is transcribed, points to the suggestion that nuc-ErbB3 is a result of alternative transcription initiation. Additional verification for the existence of nuc-ErbB3 was obtained using Schwann cell mRNA Northern blots and tissue-specific mRNA Northern (Fig. 3 ), whereas cloning and expression of nuc-ErbB3 proved that this ErbB3 variant encodes a nuclear $\sim 50 \mathrm{kDa}$ protein. Recent reports showing the presence of a $50-55 \mathrm{kDa}$ ErbB3 protein that translocates to the nucleus in both juvenile and adult monkey brain suggest the possible existence of a nuclear ErbB3 variant throughout the vertebrate nervous system (Thompson et al., 2007). Finally, the developmental expression profile of nuc-ErbB3 in rat sciatic nerves suggests a tight regulation of nuc-ErbB3 expression 
in relation to the myelination program in peripheral nerves in vivo. Regulation of spatial and temporal expression of the ErbB3 variants may play a critical role in coordinating ErbB3 function during and after development especially in disease and injury response.

In recent years, several groups have tried to elucidate the function of NRG1-ErbB2/3 axis in Schwann cells through the generation of genetically modified mice. The first series of mice lacking NRG1, ErbB2, or ErbB3 established the essential role of these molecules in Schwann cell precursor development (Lee et al., 1995; Meyer and Birchmeier, 1995; Erickson et al., 1997; Riethmacher et al., 1997). However, these mice die as embryos because of cardiac defects; thus, these initial studies were limited to early Schwann cell development. Subsequently, ErbB-receptor knockouts, which were rescued by expression of ErbB2 receptors in the heart (Morris et al., 1999; Woldeyesus et al., 1999), addressed the importance of the NRG1-ErbB2/3 axis in the development, maturation, and maintenance of Schwann cells and the peripheral nervous system. However, none of these studies addressed the existence and role of nuc-ErbB3 in Schwann cells. One may argue that this suggests that nuc-ErbB3 is not a significant component of the NRG1-ErbB3 axis, but we believe the contrary for several reasons. First, knocking out the full-length receptor may also affect the generation of the alternatively generated variants through relocation of the splicing sites or frame shifting. Second and most important, the levels and correct timing of nuc-ErbB3 expression may be regulated by the NRG1-ErbB3 signaling as part of a feedback regulatory mechanism in Schwann cells, which means that disruption of the NRG1-ErbB3 axis will affect the expression and function of nuc-ErbB3. In fact, our experiments regarding the regulatory role of neuregulin on nuc-ErbB3 translation and the developmental profile of nuc-ErbB3 suggest that the neuregulin-ErbB3 signaling axis controls the expression of nuc-ErbB3 in Schwann cells. To our knowledge, this is the first indication that a RTK controls alternative transcription from its own gene through signaling that originates from the interaction with its cognate ligand.

Glial cell proliferation and migration along the axons involves signaling pathways that may be cell type and context dependent. This includes almost all aspects of cell biology, including cytoskeletal organization, membrane trafficking, cell proliferation, migration, adhesion, and establishment of cell polarity. An indication of the role of ErbB2-ErbB3 signaling during the initial events of Schwann cell migration along the axons came from ErbB2 mutant mice in which Schwann cells are completely absent from peripheral nerves. Although Schwann cell precursors are present in the dorsal root ganglia, the migration of Schwann cells is impaired because of the loss of ErbB2-ErbB3-neuregulin axis (Morris et al., 1999). In this context, we present a significant finding that nuc-ErbB3 associates with genomic regions, which include promoters regulating the expression of various proteins that play a role in coordinating the complex processes of migration, radial sorting of axons, ensheathment, and myelination (e.g., ezrin, RhoGDI, plastin) (supplemental Table 2, available at www.jneurosci.org as supplemental material). Furthermore, nuc-ErbB3 regulates the promoter activity of ezrin in Schwann cells. This interaction may be direct through a nuc-ErbB3-DNA binding or indirect through interaction of nuc-ErbB3 with a nuclear cofactor. The fact that the appearance of ezrin in the nascent nodes precedes compact myelin formation (Melendez-Vasquez et al., 2001) suggests that the effects of the inhibition of nucErbB3 expression on the distribution of ezrin at the nodes takes place before myelination. However, we cannot exclude the pos- sibility that, since inhibition of nuc-ErbB3 expression reduces myelination, this by itself influences ezrin nodal clustering. Our results regarding the effect of nuc-ErbB3 in the rate of myelination further suggest a complex regulatory role for nuc-ErbB3. As a nuclear protein, nuc-ErbB3 functions as a transcriptional regulator (Fig. 4), and this role can be either through direct binding to DNA or through interactions with other nuclear proteins/ transcription factors, suggesting that the observed reduction in myelination is probably the result of a network of interactions in the nucleus of Schwann cells. We would like to point to the fact that this role of nuc-ErbB3 appears to be complementary to the main role of Neuregulin-ErbB3 signaling axis for Schwann cell myelination.

Collectively, our work provides evidence for a novel mechanism of RTK-mediated regulation of cellular processes independent of the classical paradigm of RTK signaling or the Notch-type cleavage signaling pathways. Nuclear expression through alternative transcription initiation might be a general feature of many membrane RTKs. In addition to spatiotemporal regulation of the expression of receptors and ligands, the subcellular compartmentalization of receptor subdomains may contribute to the finetuning of gene regulation. Moreover, the expression of ErbB3 in the nucleus of human prostate (Koumakpayi et al., 2006, 2007) and mammary (Offterdinger et al., 2002) cancer cells has been documented. In this context, the perturbation of RTK nuclear variant expression might be implicated in neuronal pathologies as well as the malignant transformation and progression of various cancers. Understanding the mechanisms of regulation of RTK nuclear expression may lead to the design of better interventional strategies addressing the initial events of cell interactions in physiology and disease.

\section{References}

Ahmad S, Gromiha MM, Sarai A (2004) Analysis and prediction of DNAbinding proteins and their binding residues based on composition, sequence and structural information. Bioinformatics 20:477-486.

Bednenko J, Cingolani G, Gerace L (2003) Nucleocytoplasmic transport: navigating the channel. Traffic 4:127-135.

Berryman M, Franck Z, Bretscher A (1993) Ezrin is concentrated in the apical microvilli of a wide variety of epithelial cells whereas moesin is found primarily in endothelial cells. J Cell Sci 105:1025-1043.

Cao X, Südhof TC (2001) A transcriptionally [correction of transcriptively] active complex of APP with Fe65 and histone acetyltransferase Tip60. Science 293:115-120.

Carey DJ, Todd MS (1987) Schwann cell myelination in a chemically defined medium: demonstration of a requirement for additives that promote Schwann cell extracellular matrix formation. Brain Res 429:95-102.

Carpenter G (2003) Nuclear localization and possible functions of receptor tyrosine kinases. Curr Opin Cell Biol 15:143-148.

Chen N, Ye XC, Chu K, Navone NM, Sage EH, Yu-Lee LY, Logothetis CJ, Lin SH (2007) A secreted isoform of ErbB3 promotes osteonectin expression in bone and enhances the invasiveness of prostate cancer cells. Cancer Res 67:6544-6548.

Citri A, Skaria KB, Yarden Y (2003) The deaf and the dumb: the biology of ErbB-2 and ErbB-3. Exp Cell Res 284:54-65.

Cokol M, Nair R, Rost B (2000) Finding nuclear localization signals. EMBO Rep 1:411-415.

Corfas G, Rosen KM, Aratake H, Krauss R, Fischbach GD (1995) Differential expression of ARIA isoforms in the rat brain. Neuron 14:103-115.

Davies AM (1998) Neuronal survival: early dependence on Schwann cells. Curr Biol 8:R15-R18.

Dennis G Jr, Sherman BT, Hosack DA, Yang J, Gao W, Lane HC, Lempicki RA (2003) DAVID: Database for Annotation, Visualization, and Integrated Discovery. Genome Biol 4:P3.

Dong Z, Brennan A, Liu N, Yarden Y, Lefkowitz G, Mirsky R, Jessen KR (1995) Neu differentiation factor is a neuron-glia signal and regulates survival, proliferation, and maturation of rat Schwann cell precursors. Neuron 15:585-596. 
Einheber S, Milner TA, Giancotti F, Salzer JL (1993) Axonal regulation of Schwann cell integrin expression suggests a role for alpha 6 beta 4 in myelination. J Cell Biol 123:1223-1236.

Elenius K, Corfas G, Paul S, Choi CJ, Rio C, Plowman GD, Klagsbrun M (1997) A novel juxtamembrane domain isoform of HER4/ErbB4. Isoform-specific tissue distribution and differential processing in response to phorbol ester. J Biol Chem 272:26761-26768.

Erickson SL, O'Shea KS, Ghaboosi N, Loverro L, Frantz G, Bauer M, Lu LH, Moore MW (1997) ErbB3 is required for normal cerebellar and cardiac development: a comparison with ErbB2-and heregulin-deficient mice. Development 124:4999-5011.

Frade JM (2005) Nuclear translocation of the p75 neurotrophin receptor cytoplasmic domain in response to neurotrophin binding. J Neurosci 25:1407-1411.

Garratt AN, Britsch S, Birchmeier C (2000) Neuregulin, a factor with many functions in the life of a Schwann cell. Bioessays 22:987-996.

Ishigaki Y, Li X, Serin G, Maquat LE (2001) Evidence for a pioneer round of mRNA translation: mRNAs subject to nonsense-mediated decay in mammalian cells are bound by CBP80 and CBP20. Cell 106:607-617.

Koumakpayi IH, Diallo JS, Le Page C, Lessard L, Gleave M, Bégin LR, MesMasson AM, Saad F (2006) Expression and nuclear localization of ErbB3 in prostate cancer. Clin Cancer Res 12:2730-2737.

Koumakpayi IH, Diallo JS, Le Page C, Lessard L, Filali-Mouhim A, Bégin LR, Mes-Masson AM, Saad F (2007) Low nuclear ErbB3 predicts biochemical recurrence in patients with prostate cancer. BJU Int 100:303-309.

Lallemand D, Manent J, Couvelard A, Watilliaux A, Siena M, Chareyre F, Lampin A, Niwa-Kawakita M, Kalamarides M, Giovannini M (2009) Merlin regulates transmembrane receptor accumulation and signaling at the plasma membrane in primary mouse Schwann cells and in human schwannomas. Oncogene 28:854-865.

Lee HJ, Jung KM, Huang YZ, Bennett LB, Lee JS, Mei L, Kim TW (2002) Presenilin-dependent gamma-secretase-like intramembrane cleavage of ErbB4. J Biol Chem 277:6318-6323.

Lee KF, Simon H, Chen H, Bates B, Hung MC, Hauser C (1995) Requirement for neuregulin receptor erbB2 in neural and cardiac development. Nature 378:394-398.

Levi AD, Bunge RP, Lofgren JA, Meima L, Hefti F, Nikolics K, Sliwkowski MX (1995) The influence of heregulins on human Schwann cell proliferation. J Neurosci 15:1329-1340.

Li W, Jaroszewski L, Godzik A (2001) Clustering of highly homologous sequences to reduce the size of large protein databases. Bioinformatics 17:282-283.

Maquat LE (1995) When cells stop making sense: effects of nonsense codons on RNA metabolism in vertebrate cells. RNA 1:453-465.

Maquat LE, Carmichael GG (2001) Quality control of mRNA function. Cell 104:173-176.

Melendez-Vasquez CV, Rios JC, Zanazzi G, Lambert S, Bretscher A, Salzer JL (2001) Nodes of Ranvier form in association with ezrin-radixin-moesin (ERM)-positive Schwann cell processes. Proc Natl Acad Sci U S A 98:1235-1240.

Meyer D, Birchmeier C (1995) Multiple essential functions of neuregulin in development. Nature 378:386-390.

Meyer D, Yamaai T, Garratt A, Riethmacher-Sonnenberg E, Kane D, Theill LE, Birchmeier C (1997) Isoform-specific expression and function of neuregulin. Development 124:3575-3586.

Michailov GV, Sereda MW, Brinkmann BG, Fischer TM, Haug B, Birchmeier C, Role L, Lai C, Schwab MH, Nave KA (2004) Axonal neuregulin-1 regulates myelin sheath thickness. Science 304:700-703.

Morris JK, Lin W, Hauser C, Marchuk Y, Getman D, Lee KF (1999) Rescue of the cardiac defect in ErbB2 mutant mice reveals essential roles of ErbB2 in peripheral nervous system development. Neuron 23:273-283.

Morrissey TK, Levi AD, Nuijens A, Sliwkowski MX, Bunge RP (1995) Axon- induced mitogenesis of human Schwann cells involves heregulin and p185erbB2. Proc Natl Acad Sci U S A 92:1431-1435.

Muraoka-Cook RS, Sandahl M, Husted C, Hunter D, Miraglia L, Feng SM, Elenius K, Earp HS 3rd (2006) The intracellular domain of ErbB4 induces differentiation of mammary epithelial cells. Mol Biol Cell 17:4118-4129

Ni CY, Murphy MP, Golde TE, Carpenter G (2001) gamma-Secretase cleavage and nuclear localization of ErbB-4 receptor tyrosine kinase. Science 294:2179-2181.

Offterdinger M, Schöfer C, Weipoltshammer K, Grunt TW (2002) c-erbB-3: a nuclear protein in mammary epithelial cells. J Cell Biol 157:929-939.

Prigent SA, Lemoine NR, Hughes CM, Plowman GD, Selden C, Gullick WJ (1992) Expression of the c-erbB-3 protein in normal human adult and fetal tissues. Oncogene 7:1273-1278.

Raabe TD, Deadwyler G, Varga JW, Devries GH (2004) Localization of neuregulin isoforms and erbB receptors in myelinating glial cells. Glia 45:197-207.

Riethmacher D, Sonnenberg-Riethmacher E, Brinkmann V, Yamaai T, Lewin GR, Birchmeier C (1997) Severe neuropathies in mice with targeted mutations in the ErbB3 receptor. Nature 389:725-730.

Sardi SP, Murtie J, Koirala S, Patten BA, Corfas G (2006) Presenilindependent ErbB4 nuclear signaling regulates the timing of astrogenesis in the developing brain. Cell 127:185-197.

Scherer SS, Xu T, Crino P, Arroyo EJ, Gutmann DH (2001) Ezrin, radixin, and moesin are components of Schwann cell microvilli. J Neurosci Res 65:150-164.

Steiner H, Haass C (2000) Intramembrane proteolysis by presenilins. Nat Rev Mol Cell Biol 1:217-224.

Sweet DJ, Gerace L (1995) Taking from the cytoplasm and giving to the pore: soluble transport factors in nuclear protein import. Trends Cell Biol 5:444-447.

Syroid DE, Maycox PR, Burrola PG, Liu N, Wen D, Lee KF, Lemke G, Kilpatrick TJ (1996) Cell death in the Schwann cell lineage and its regulation by neuregulin. Proc Natl Acad Sci U S A 93:9229-9234.

Taveggia C, Zanazzi G, Petrylak A, Yano H, Rosenbluth J, Einheber S, Xu X, Esper RM, Loeb JA, Shrager P, Chao MV, Falls DL, Role L, Salzer JL (2005) Neuregulin-1 type III determines the ensheathment fate of axons. Neuron 47:681-694.

Thompson M, Lauderdale S, Webster MJ, Chong VZ, McClintock B, Saunders R, Weickert CS (2007) Widespread expression of ErbB2, ErbB3 and ErbB4 in non-human primate brain. Brain Res 1139:95-109.

Vecchi M, Carpenter G (1997) Constitutive proteolysis of the ErbB-4 receptor tyrosine kinase by a unique, sequential mechanism. J Cell Biol 139:995-1003.

Weinmann AS, Farnham PJ (2002) Identification of unknown target genes of human transcription factors using chromatin immunoprecipitation. Methods 26:37-47.

Windebank AJ, Wood P, Bunge RP, Dyck PJ (1985) Myelination determines the caliber of dorsal root ganglion neurons in culture. J Neurosci 5:1563-1569.

Woldeyesus MT, Britsch S, Riethmacher D, Xu L, Sonnenberg-Riethmacher E, Abou-Rebyeh F, Harvey R, Caroni P, Birchmeier C (1999) Peripheral nervous system defects in erbB2 mutants following genetic rescue of heart development. Genes Dev 13:2538-2548.

Xu L, Massagué J (2004) Nucleocytoplasmic shuttling of signal transducers. Nat Rev Mol Cell Biol 5:209-219.

Xue C, Liang F, Mahmood R, Vuolo M, Wyckoff J, Qian H, Tsai KL, Kim M, Locker J, Zhang ZY, Segall JE (2006) ErbB3-dependent motility and intravasation in breast cancer metastasis. Cancer Res 66:1418-1426.

Yamauchi J, Miyamoto Y, Chan JR, Tanoue A (2008) ErbB2 directly activates the exchange factor Dock7 to promote Schwann cell migration. J Cell Biol 181:351-365. 